\title{
Experimental repetitive quantum channel simulation
}

\author{
L. Hu, ${ }^{1, *}$ X. Mu, ${ }^{1, *}$ W. Cai, ${ }^{1}$ Y. Ma,${ }^{1}$ Y. Xu,,${ }^{1}$ H. Wang,,${ }^{1}$ Y. P. Song,,${ }^{1}$ C.-L. Zou,${ }^{2, \dagger}$ and L. Sun ${ }^{1,+}$ \\ ${ }^{1}$ Center for Quantum Information, Institute for Interdisciplinary Information Sciences, Tsinghua University, Beijing 100084, China \\ ${ }^{2}$ Key Laboratory of Quantum Information, CAS, University of Science and Technology of China, Hefei, Anhui 230026, P. R. China
}

\begin{abstract}
Universal control of quantum systems is a major goal to be achieved for quantum information processing, which demands thorough understanding of fundamental quantum mechanics and promises applications of quantum technologies. So far, most studies concentrate on ideally isolated quantum systems governed by unitary evolutions, while practical quantum systems are open and described by quantum channels due to their inevitable coupling to environment. Here, we experimentally simulate arbitrary quantum channels for an open quantum system, i.e. a single photonic qubit in a superconducting quantum circuit. The arbitrary channel simulation is achieved with minimum resource of only one ancilla qubit and measurement-based adaptive control. By repetitively implementing the quantum channel simulation, we realize an arbitrary Liouvillian for a continuous evolution of an open quantum system for the first time. Our experiment provides not only a testbed for understanding quantum noise and decoherence, but also a powerful tool for full control of practical open quantum systems.
\end{abstract}

\section{INTRODUCTION}

Experimental quantum systems are open in reality, since they are inevitably coupled to environment. Therefore, the real physical effect on a quantum state should be a completely positive and trace-preserving mapping, also called quantum channel, instead of a unitary evolution for an ideally isolated quantum system [1-3]. Therefore, the simulation of an arbitrary quantum channel not only is of fundamental importance for the understanding of quantum noise and decoherence, but also allows for applications which rely on universal control of open quantum systems. For example, quantum channel could be used for the preparation of arbitrarily mixed quantum state, which would be a resource for fundamental quantum mechanics and for deterministic quantum computation with one qubit $[4,5]$. A continuous quantum channel can be utilized for realizing quantum state stabilization [6] and autonomous quantum error correction via the quantum Zeno effect [7-9]. There are also appealing proposals using quantum channels for studying non-equilibrium quantum systems $[10,11]$ and preparing topological quantum many-body states [12].

For an open quantum system constantly coupled to its environment, the quantum state evolution follows a Liouvillian of master equation [2,13]. A natural choice to simulate such a quantum channel is to construct an artificial environment and engineer proper system-environment interaction $[14,15]$. For example, the damping channel of a qubit can be realized by coupling it to a resonator with low quality factor [16]. Rather than such an analog approach, it is more attractive to realize a universal digital quantum channel simulation (QCS) through a standard quantum circuit with continuous Liouvillians which can be realized piecewisely by repeating a quantum channel. The digital QCS can be straightforwardly implemented based on Stinespring's dilation with a unitary operation on the expanded Hilbert space including both the target quantum system and the environment followed by discarding the environment in the end. Although proof-of-principle experiments have been reported in various quantum comput- ing platforms $[17,18]$, such an approach is not scalable since the required dimension of the ancilla and number of multiqubit gate operations scale polynomially with the target system dimension [19]. Recently, a convenient approach to realize arbitrary qubit channel with minimum ancillary resource is proposed in Ref. 20, which relies on decomposing the channel into convex combination of quasiextreme channels. Such an approach has been demonstrated experimentally [21, 22]. However, their simulation is probabilistic, and repetitive implementation is very challenging.

Here we deterministically implement repetitive singlequbit digital QCS within a superconducting system with a circuit quantum electrodynamics (cQED) architecture [2325]. The digital QCS benefits from the high-fidelity quantum non-demolition (QND) measurement of superconducting qubits and fast real-time adaptive control based on field programmable gate arrays (FPGA). We first realize two typical channels of dephasing and amplitude damping for a system qubit, and demonstrate the controlling of external dephasing and damping rates in a large range (2-3 orders of magnitude) respectively. Furthermore, we realize arbitrary QCS based on the proposal in Ref. 20, which yields an average state generation fidelity of $97 \%$ and is mainly limited by the decoherence of the ancilla qubit. The repetitive QCS with controllable parameters provides a testbed for studying reservoir engineering [6], quantum Zeno effect [7-9, 26], quantum thermodynamics [27, 28], and quantum metrology [29, 30]. Together with the recently demonstrated arbitrary unitary control and quantum error correction [31,32] in cQED architecture, our demonstrated QCS could realize reliable universal control of open quantum system, which is significant for quantum computation $[1,25]$ and simulation $[33,34]$.

\section{RESULTS}

\section{A. Principle and system}

Figure 1(a) is a schematic of the time evolution of an open quantum system coupled to a reservoir of harmonic oscil- 


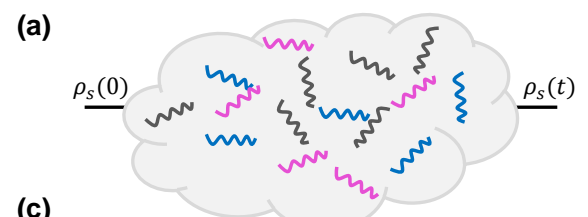

(c)

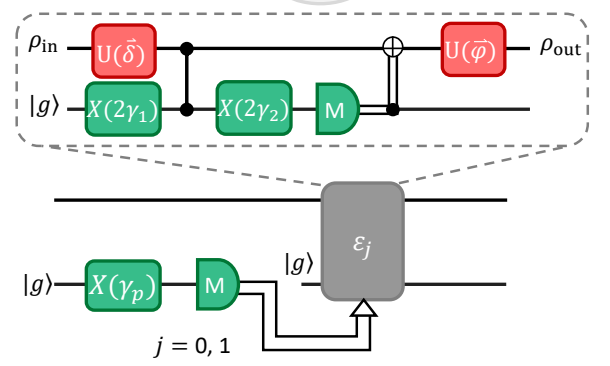

(b)

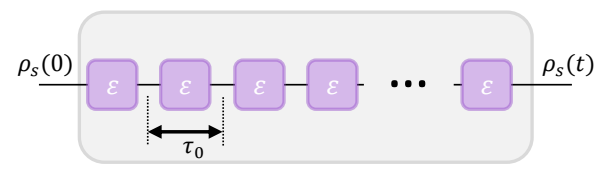

(d)

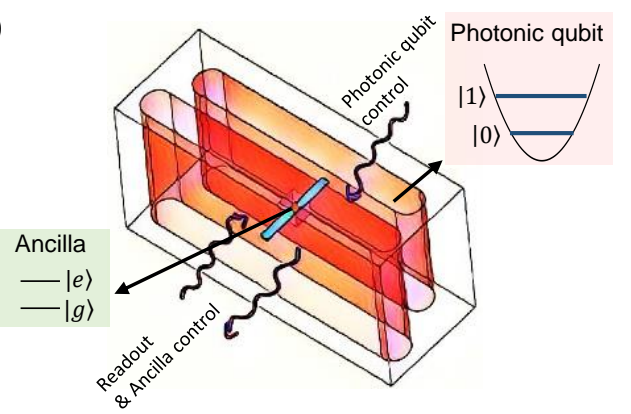

FIG. 1. The schematic of quantum channel simulation. (a) Evolution of an open quantum system coupled to a bath of harmonic oscillators. (b) Evolution of a quantum system under repetitive quantum channel operation $\varepsilon$ with a time interval $\tau_{0}$. (c) Quantum circuit for realizing arbitrary single-qubit quantum channels with the assistance of one ancilla qubit. $U$ is a unitary operation on the system qubit. $X(\gamma)$ represents a rotation of the ancilla qubit along $X$-axis with an angle $\gamma . M$ denotes a measurement on the ancilla qubit. (d) Experimental system of a three-dimensional cQED architecture for repetitive QCS. Fock states $\{|0\rangle,|1\rangle\}$ in a microwave cavity constitute the photonic qubit on which channel simulations are performed, while a transmon qubit with states $\{|g\rangle,|e\rangle\}$ serves as an ancilla.

lators. Due to the inaccessibility of the environment, the evolution of the system is described by a quantum channel $\mathscr{E}_{t}: \rho_{\mathrm{s}}(0) \mapsto \rho_{\mathrm{s}}(t)$, instead of unitary operations for a closed quantum system. For a typical open quantum system with time-independent system-environment interaction, the Hamiltonian under the rotating-wave approximation reads $H_{\mathrm{se}}=\int d \omega\left(g_{\omega} o^{\dagger} b_{\omega}+h . c.\right)$, where $b_{\omega}$ is the bosonic operator associated with the environment degrees of freedom, $o$ is the jump operator of the system, and $g_{\omega}$ is the interaction strength. Applying the Born-Markov approximation, the evolution of the system or the quantum channel follows the master equation, and the dynamics of quantum states satisfy [Supplementary Information]

$$
\rho_{\mathrm{S}}(t)=e^{t \mathscr{L}} \rho_{\mathrm{S}}(0),
$$

with the Lindblad form of the Liouvillian as [13]

$$
\mathscr{L} \rho=\sum \kappa\left(2 o \rho o^{\dagger}-o^{\dagger} o \rho-\rho o^{\dagger} o\right) .
$$

Here, $\kappa$ is the decoherence rate determined by $g_{\omega}$ and the density of states of the environment.

Alternatively, the quantum channel can be represented in the Kraus representation as [1]

$$
\rho_{\mathrm{s}}(t)=\mathscr{E}_{t}\left[\rho_{\mathrm{s}}(0)\right]=\sum_{k} E_{k}^{(t)} \rho_{\mathrm{s}}(0) E_{k}^{(t) \dagger}
$$

with the Kraus operators satisfying $\sum_{k} E_{k}^{(t) \dagger} E_{k}^{(t)}=\mathbb{I}$. This formula describes a discrete mapping between quantum states at different times.

According to the two descriptions of quantum channels, there are different approaches to simulate a quantum channel. It is straightforward to simulate the continuous dynamics of an open quantum system through an analog approach, in which a given Liouvillian can be realized by directly constructing an environment with certain density of states and engineering the proper system-environment interaction. Another approach is piecewise implementation of a channel $\mathscr{E}_{\tau_{0}}$ by a standard quantum circuit with an interval $\tau_{0}$, as shown in Fig. 1(b). The dynamics of the open quantum system can be digitally simulated as $\mathscr{E}_{t}=\left[\mathscr{E}_{\tau_{0}}\right]^{t / \tau_{0}}: \rho_{\mathrm{s}}(0) \mapsto \rho_{\mathrm{s}}(t)$. This digital approach is universal because arbitrary continuous evolution of a quantum system in time can be realized by repetitively implementing QCS. For instance, the Liouvillian of Eq. (2) can be simulated digitally in the limit of $\kappa \tau_{0} \ll 1$ (Supplementary Information, also see [35]), with

$$
\begin{aligned}
& E_{0}^{\left(\tau_{0}\right)}=\sqrt{2 \kappa \tau_{0}} o, \\
& E_{1}^{\left(\tau_{0}\right)}=\mathbb{I}-\kappa \tau_{0} o^{\dagger} o .
\end{aligned}
$$

Although the Markovian environment contains huge degrees of freedom, as proved theoretically $[20,36]$ an arbitrary quantum channel for a system qubit can be efficiently and deterministically simulated with minimum resource of one single ancilla qubit and measurement-based adaptive control. The quantum circuit for an arbitrary qubit QCS is shown in Fig. 1(c). As a "quantum dice", when the ancilla qubit (initially in the ground state $|g\rangle$ ) is tossed by rotating it along $X$-axis with an angle $\gamma_{p}$ and then measuring it in the computational basis $\{|g\rangle,|e\rangle\}$, a random bit $j \in\{0,1\}$ is generated with the probabilities $\left\{\cos ^{2} \frac{\gamma_{p}}{2}, \sin ^{2} \frac{\gamma_{p}}{2}\right\}$. By randomly choosing the quantum circuit $\mathscr{E}_{j}$ (a quasiextreme channel) according to the result of the toss, an arbitrary qubit channel can be obtained deterministically as a convex combination of these two quasiextreme channels [20]. After the toss, the ancilla qubit is also utilized in the quasiextreme channel simulations [Fig. 1(c)] to assist the unitary operations on the system qubit, e.g., implementing a controlled-phase (CZ) gate and 
(a)
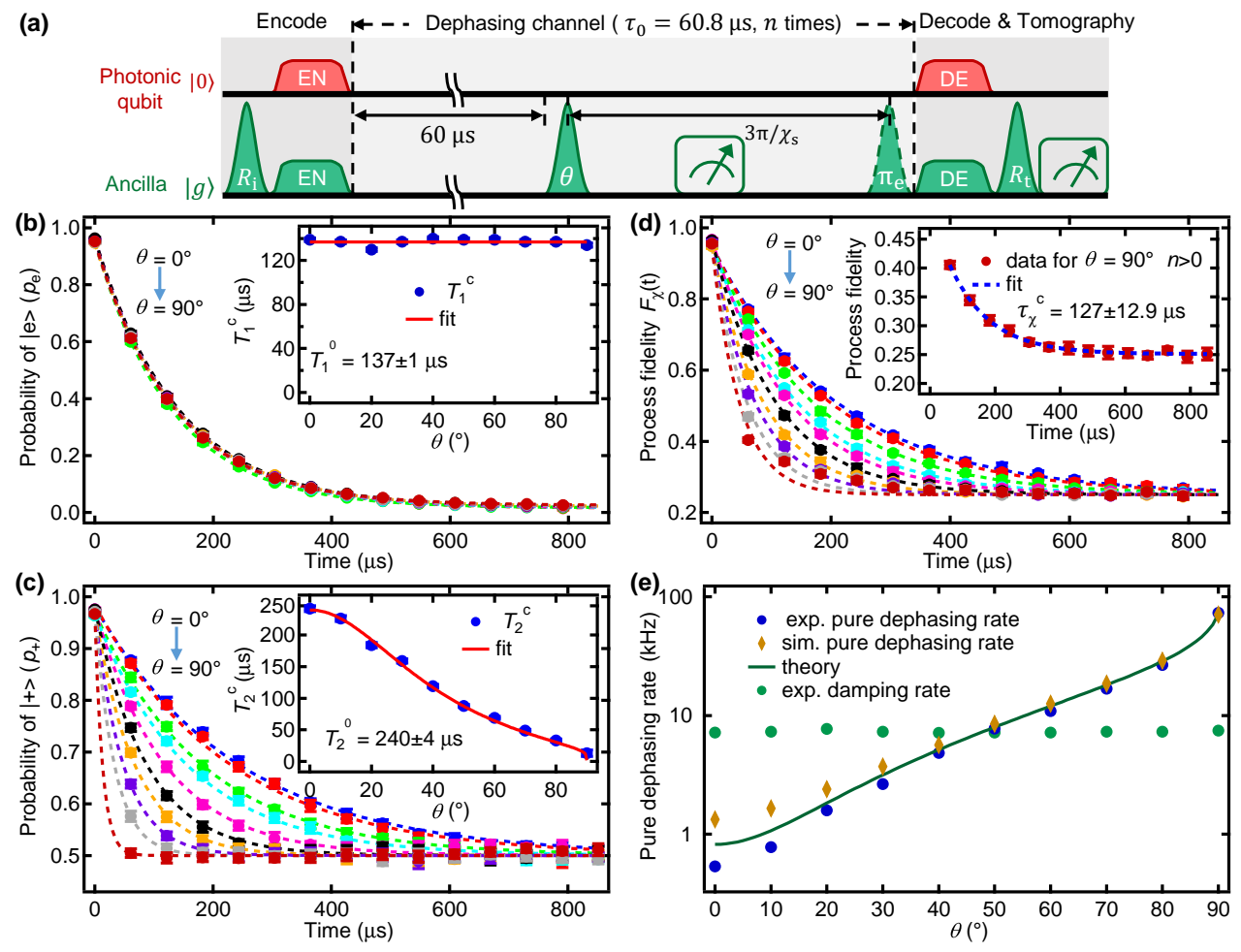

FIG. 2. Repetitive single-qubit dephasing channel simulation. (a) Experimental sequence. In each experiment, the initialization and encoding processes are performed at the very beginning, and the quantum channel (consisting of two operations on the ancilla qubit and a controlled-phase gate between the ancilla and the photonic qubit over a time interval of $3 \pi / \chi_{\mathrm{s}}$ ) is repeated $n$ times. Decoding and tomography are performed in the end to characterize the channel performance. (b) and (c) The longitudinal and transverse relaxation of the photonic qubit with repetitive QCS. Insets: the extracted $T_{1}^{\mathrm{c}}$ and $T_{2}^{\mathrm{c}}$ against the control parameter $\theta$ of the channel. $T_{1}^{\mathrm{c}}$ curves are fitted with $1 / T_{1}^{\mathrm{c}}=1 / T_{1}{ }^{0}$ while $T_{2}^{\mathrm{c}}$ curves are fitted with $1 / T_{2}^{\mathrm{c}}=-\ln \left(\left[2 \cos ^{2}(\theta / 2)-1\right]\right) / \tau_{0}+1 / T_{2}{ }^{0}$, where $T_{1}^{0}$ and $T_{2}^{0}$ are free parameters and approach the intrinsic decoherence times $T_{1}^{\mathrm{s}}$ and $T_{2}^{\mathrm{s}}$ of the photonic qubit. (d) The decay of channel process fidelity $F_{\chi}$ with different $\theta$. (e) The pure dephasing rate is controlled over a range of two orders of magnitude by varying $\theta$, in good agreement with theoretical expectation and simulation.

measurement-based adaptive control as in our experiments.

As schematically shown in Fig. 1(d), our experimental device consists of a superconducting transmon qubit dispersively coupled to two waveguide cavity resonators [24, 32, 37-40]. One of the cavities (storage cavity) has long photon coherence times $T_{1}^{\mathrm{s}}=143 \mu \mathrm{s}$ and $T_{2}^{\mathrm{s}}=250 \mu \mathrm{s}$, and its $|0\rangle$ and $|1\rangle$ Fock states constitute the two bases of a photonic qubit (the system qubit) on which the QCS are performed. The transmon qubit as an ancilla with an energy relaxation time $T_{1}=30 \mu \mathrm{s}$ and a pure dephasing time $T_{\varphi}=120 \mu \mathrm{s}$ is used to realize the QCS. The other short-lived cavity with a photon lifetime $\tau_{\mathrm{r}}=44 \mathrm{~ns}$ is to readout the ancilla qubit with the help of a phase-sensitive Josephson bifurcation amplifier [41-44] for a high fidelity single-shot QND measurement. Each readout measurement throughout our experiment returns a digitized value of the qubit state $(0$ and 1 correspond to the ground state $|g\rangle$ and excited state $|e\rangle$, respectively). Fast real-time adaptive control is also vital for the demonstrated QCS. This is achieved through three FPGAs with home-made logics, which allow us to individually control the ancilla qubit, the photonic qubit, and the readout cavity, and also integrate readout signal sampling, ancilla state estimation, and manipulation signal generation together. The experimental appara- tus and readout properties are similar to the earlier report in Ref. 32.

The ancilla qubit and storage cavity are well described by the dispersive Hamiltonian (The readout cavity has been neglected since it remains in vacuum unless a measurement is performed)

$$
H / \hbar=\omega_{s} a^{\dagger} a+\omega_{a}|e\rangle\left\langle e\left|-\chi_{\mathrm{s}} a^{\dagger} a\right| e\right\rangle\langle e|
$$

where $a^{\dagger}(a)$ is the creation (annihilation) operator of the storage cavity, $|e\rangle$ is the excited state of the ancilla qubit, and $\chi_{\mathrm{s}} / 2 \pi=1.90 \mathrm{MHz}$ is the dispersive interaction strength between the qubit and the storage cavity. This strong dispersive coupling gives rise to the necessary operations for the simulation, including unitary operations on the photonic qubit and the two-qubit gates. The control pulses are numerically optimized with the gradient ascent pulse engineering (GRAPE) method $[45,46]$ based on carefully calibrated experimental parameters. 

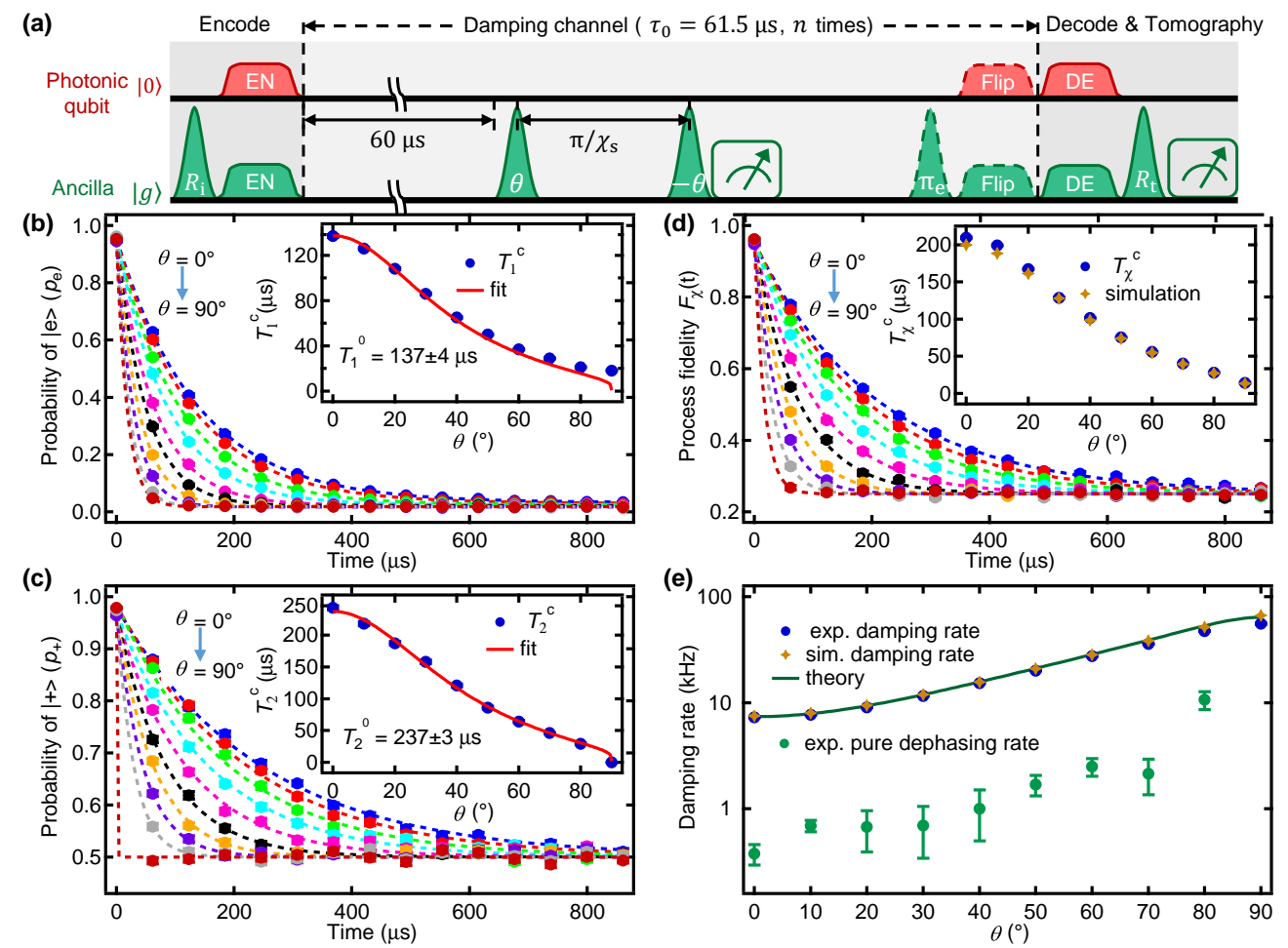

FIG. 3. Repetitive single-qubit damping channel simulation. (a) Experimental sequence. (b) and (c) The longitudinal and transverse relaxation of the photonic qubit with repetitive QCS. Insets: the extracted $T_{1}^{\mathrm{c}}$ and $T_{2}^{\mathrm{c}}$ against the control parameter $\theta$ of the channel. $T_{1}^{\mathrm{c}}$ curves are fitted with $1 / T_{1}^{\mathrm{c}}=-\ln \left(\cos ^{2} \theta\right) / \tau_{0}+1 / T_{1}{ }^{0}$, resulting in $T_{1}^{0}=137 \mu \mathrm{s}$. $T_{2}^{\mathrm{c}}$ curves are fitted with $1 / T_{2}^{\mathrm{c}}=-\ln \left(\cos ^{2} \theta\right) / 2 \tau_{0}+1 / T_{2}{ }^{0}$, resulting in $T_{2}{ }^{0}=237 \mu$ s. (d) The decay of channel process fidelity $F_{\chi}$ with different $\theta$. (e) The damping and pure dephasing rates versus $\theta$, obtained from experimental results, simulation, and theoretical prediction, respectively.

\section{B. Qubit dephasing channel}

To illustrate the concept of ancilla-assisted qubit QCS, we start with the simple dephasing channel simulation for the photonic qubit. As shown by the experimental pulse sequence in Fig. 2(a), the system is initialized to $|0\rangle \otimes|g\rangle$, and the photonic qubit is prepared to a pure state with the assistance of the ancilla, while the ancilla goes back to $|g\rangle$ after the encoding process. Then, the dephasing channel is implemented through three steps: (i) state preparation of the ancilla qubit by $X_{\theta}$, i.e., a rotation of the ancilla with an angle $\theta$ along the $X$-axis. (ii) $\mathrm{CZ}$ gate induced by the dispersive interaction between the ancilla and the photonic qubit over a time interval of $3 \pi / \chi_{\mathrm{s}}$. (iii) a projective measurement followed by a conditional gate on the ancilla qubit to reset it to $|g\rangle$, thus erasing the quantum information on the ancilla. During the channel simulation, there is no external operation on the photonic qubit. Therefore, the population of the photonic qubit is not changed by the channel and only the phase of the photonic qubit is entangled with the ancilla that induces the dephasing process. Thus, the quantum channel $\mathscr{E}_{\theta}^{\mathrm{dph}}: \rho_{\mathrm{s}} \mapsto p_{\theta} \rho_{\mathrm{s}}+\left(1-p_{\theta}\right) \mathbf{Z} \rho_{\mathrm{s}} \mathbf{Z}$ is realized, where $p_{\theta}=\cos ^{2} \frac{\theta}{2}$ and $\mathbf{Z}$ is the Pauli matrix. The dephasing channel can be implemented deterministically without postselection and also without destroying the photonic qubit. The channel simulation therefore can be repeated $n$ times with a controlled repetition interval $\tau_{0}$ and dephasing probability $p_{\theta}$ to simulate an open quantum system with arbitrary dephasing rate $\kappa=-\ln \left(2 p_{\theta}-1\right) / \tau_{0}$. Finally, the information is decoded back from the photonic qubit to the ancilla with a state tomography for an evaluation of the channel performance.

In the experiment presented in Fig. 2, we fix the repetition interval $\tau_{0} \approx 61 \mu$ s while changing $\theta$, i.e., the dephasing rate continuously. Figures $2(\mathrm{~b})$ and $2(\mathrm{c})$ show the measured probability $p_{e}=\left\langle e\left|\left(\mathscr{E}_{\theta}^{\mathrm{dph}}\right)^{n}\left(\rho_{\text {in }}\right)\right| e\right\rangle$ and $p_{+}=$ $\left\langle+\left|\left(\mathscr{E}_{\theta} \mathrm{dph}\right)^{n}\left(\rho_{\text {in }}\right)\right|+\right\rangle$ with the initial state of $\rho_{\text {in }}=|1\rangle\langle 1|$ and $|+\rangle\langle+|$, respectively. Here, $|+\rangle=(|g\rangle+|e\rangle) / \sqrt{2}$. The results correspond to the longitudinal and transverse relaxation of the photonic qubit induced by the channel, from which we can extract $T_{1}^{\mathrm{c}}$ and $T_{2}^{\mathrm{c}}$ of the simulated channel coherence times for different $\theta$. As expected, $T_{1}^{\mathrm{c}}$ is almost not affected by this dephasing channel while the effective dephasing time $T_{2}^{\mathrm{c}}$ becomes shorter for a larger $\theta$. When $\theta=0$ (no extra dephasing), we get $T_{1}^{\mathrm{c}}(0)=139 \mu \mathrm{s}$ and $T_{2}^{\mathrm{c}}(0)=242 \mu \mathrm{s}$, agreeing well with the intrinsic coherence times of the photonic qubit.

To fully characterize the channel, we also measure the process $\chi$ matrix of the channel, and calculate the process fidelity $F_{\chi}(t)=\operatorname{Tr}\left(\chi_{\mathrm{dph}} \chi_{\mathrm{I}}\right)$ with $\chi_{\mathrm{dph}}$ and $\chi_{\mathrm{I}}$ being the $\chi$ matrix of the measured dephasing channel and the identity channel, respec- 

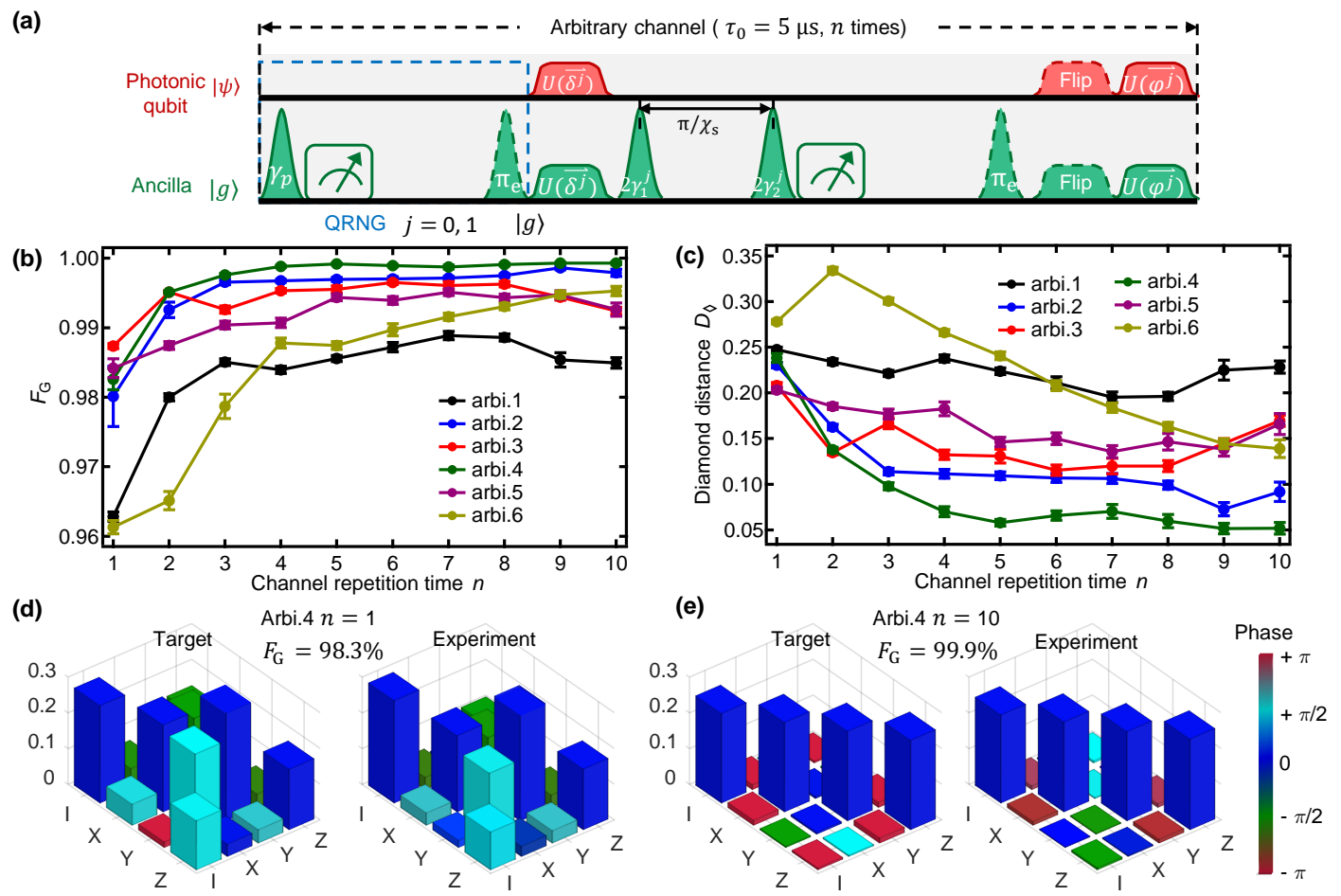

FIG. 4. Arbitrary single-qubit quantum channel simulation. (a) Pulse sequence for the arbitrary QCS protocol shown in Fig. 1(c). The QCS starts with a quantum random number generation (QRNG), realized by measuring the ancilla in a superposition state. The channel is repeated $n=1 \sim 10$ times without any time delay. (b) State generation fidelity $F_{\mathrm{G}}$ between the experimental and theoretical $\chi$ matrices as a function of $n$ for six different arbitrary channels. The simulated arbitrary channels at $n=1$ yield an average fidelity of $97 \%$. The error bars are determined by bootstrapping on the measured $\chi$ matrices. (c) Calculated diamond distance $D_{\diamond}$ between the experimental $\chi_{\mathrm{E}}$ and the target $\chi_{\mathrm{T}}$ as a function $n$ for the six arbitrary channels. (d) and (e) Typical $\chi_{\mathrm{E}}$ and $\chi_{\mathrm{T}}$ of the 4 th channel at $n=1$ and $n=10$, respectively. The height and color represent the amplitude and phase, respectively. The experiment results are in excellent agreement with theoretical expectations.

tively. As shown in Fig. 2(d), the first point $F_{\chi}(0)=95.5 \%$ indicates a high "round trip" process fidelity of the encoding and decoding processes only. Due to the large dephasing rate when $\theta$ approaches $90^{\circ}$, the photonic qubit is nearly completely dephased after the first round of QCS. This should result in $F_{\chi} \approx 0.5$. However, due to the intrinsic decoherence of the photonic qubit, $F_{\chi}$ is lower than the limit of 0.5 and eventually converges to the limit of 0.25 for a damping channel. To confirm this, a fit to the results for $n \geq 1(t>0)$ at $\theta=90^{\circ}$ is shown in Fig. 2(d) inset, which gives a decay of $127 \mu \mathrm{s}$ agreeing well with the intrinsic photon $T_{1}^{\mathrm{s}}$.

Figure 2(e) shows the experimental pure dephasing rate $\left(1 / T_{2}^{\mathrm{c}}-1 / 2 T_{1}^{\mathrm{c}}\right)$ as a function of $\theta$, in excellent agreement with theoretical expectation (see Supplementary Information) and simulation based on QuTiP in Python [47, 48] (both take the ancilla decoherence into account). As demonstrated in Fig. 2(e), the pure dephasing rate can be controlled over a range of two orders of magnitude by varying $\theta$. Since our QCS can be implemented within a duration of $\mu \mathrm{s}$, the channel repetition interval can be much shorter than $61 \mu$ s used here. The experimental results obtained with $\tau_{0}=5.8 \mu$ s are presented in Supplementary Information, and we can achieve a pure dephasing rate more than 1000 times faster than the intrinsic pure dephasing rate.

\section{Qubit amplitude damping channel}

We next implement a single-qubit amplitude damping channel, which is also a quasiextreme channel [Fig. 1(c)]. The pulse sequence is shown in [Fig. 3(a)], with the same initialization, encoding, and decoding processes as for the dephasing channel. Here, the kernel part of the damping channel is realized through a Ramsey-type of measurement with an interval of $\pi / \chi_{\mathrm{s}}$ between two rotations $X_{\theta}$ and $X_{-\theta}$. In contrast to the dephasing channel, adaptive control on the photonic qubit is necessary. If the ancilla collapses onto $|e\rangle$ after a detection, a GRAPE pulse is used to flip the photonic qubit (X gate) following a $\pi$ pulse to first reset the ancilla. The rotation angle $\theta$ determines the probability $p_{\theta}=\sin ^{2} \theta$ of the photonic qubit being flipped to $|0\rangle$ by the conditional $\mathbf{X}$ gate. Therefore, the quantum channel acting on the photonic qubit is a damping channel $\mathscr{E}_{\theta}^{\mathrm{dmp}}: \rho_{\mathrm{s}} \mapsto E_{0} \rho_{\mathrm{s}} E_{0}^{\dagger},+E_{1} \rho_{\mathrm{s}} E_{1}^{\dagger}$, with $E_{0}=|0\rangle\left\langle 0\left|+\sqrt{1-p_{\theta}}\right| 1\right\rangle\langle 1|$ and $E_{1}=\sqrt{p_{\theta}}|0\rangle\langle 1|$. By repetitively implementing the damping channel with an interval $\tau_{0}$, an effective damping rate $\kappa=-\ln \left(1-p_{\theta}\right) / \tau_{0}$ can be realized.

To compare with the dephasing channel, we fix $\tau_{0} \approx 62 \mu \mathrm{s}$ and vary $\theta$ to study the performance of the channel. From the results presented in Figs. 3(b) and 3(c), the longitudinal 
and transverse relaxation times of the channel $T_{1}^{\mathrm{c}}$ and $T_{2}^{\mathrm{c}}$ are extracted and plotted in the corresponding insets. At $\theta=0$, $T_{1}^{\mathrm{c}}(0)=137 \mu \mathrm{s}$ and $T_{2}^{\mathrm{c}}(0)=243 \mu \mathrm{s}$ approaching the intrinsic ones. When increasing $\theta$, both longitudinal and transverse relaxation times reduce, and $T_{2}^{\mathrm{c}} \approx 2 T_{1}^{\mathrm{c}}$ is satisfied for a damping channel with negligible intrinsic pure dephasing process. When $\theta$ approaches $90^{\circ}$, the photonic qubit should be completely damped to $|g\rangle$ after the first channel simulation process. However, it is noticed that the experimental $T_{1}^{\mathrm{c}}$ saturates at certain value due to the imperfections of the ancilla qubit. In Fig. 3(d), the process fidelity $F_{\chi}(t)$ is also evaluated for the damping channel, which saturates to the limit 0.25 as expected for a damping process. Figure 3(e) shows the experimental damping rate as a function of $\theta$, also in excellent agreement with theoretical expectation (see Supplementary Information) and simulation (both take the ancilla decoherence into account). This indicates a negligible pure dephasing effect from the simulated damping channel, also consistent with the nearly constant pure dephasing rate extracted from experiment.

\section{Arbitrary quantum channel simulation}

The experimental demonstrations of the dephasing and damping channels prove that our cQED platform and the adaptive control are reliable for QCS. Now, we turn to the demonstration of the most general quantum tool for arbitrary qubit QCS. The quantum circuit in Fig. 1(c) is realized with the experimental sequence in Fig. 4(a). Here, the encoding, decoding, and tomography procedures are the same as those in Fig. 2(a) and Fig. 3(a), and are omitted for simplicity. At the beginning of the QCS, the ancilla qubit is prepared in a quantum superposition state of $|g\rangle$ and $|e\rangle$, and a projective measurement on it generates a quantum random number $j \in\{0,1\}$. According to $j$, an adaptive operation on the ancilla initializes the ancilla back to $|g\rangle$, and then the $j$ th quasiextreme channel is implemented. The pulse sequences for the two quasiextreme channels are exactly the same, except that the 8 parameters $\left\{\vec{\delta}, \gamma_{1}, \gamma_{2}, \vec{\varphi}\right\}$ are different.

To simulate the arbitrary quantum channel, we first numerically generate a random quantum channel $\mathscr{E}_{\mathrm{T}}$ with the target process matrix $\chi_{\mathrm{T}}$. Using the difference between $\chi_{\mathrm{T}}$ and the expected process matrix by the quantum circuit in Fig. 1(c) as the figure of merit, the experimental parameters for the quasiextreme channels and the quantum random number are optimized to minimize the difference. We then experimentally measure the process matrix $\chi_{\mathrm{E}}$ using the optimized parameters by repeating the experimental channel $n=1 \sim 10$ times without any time delay $\left(\tau_{0}=5 \mu \mathrm{s}\right)$.

To characterize the performance of the arbitrary QCS, we use the fidelity of state generation $F_{\mathrm{G}}=\inf _{\rho} \sqrt{\sqrt{\mathscr{E}_{\mathrm{T}}(\rho)}\left[\mathscr{E}_{\mathrm{E}}(\rho)\right] \sqrt{\mathscr{E}_{\mathrm{T}}(\rho)}}$, which corresponds to the worst fidelity of the quantum state generated by the experimental channel $\mathscr{E}_{\mathrm{E}}$ (based on $\chi_{\mathrm{E}}$ ) when compared with the one by the target channel $\mathscr{E}_{\mathrm{T}}$. Figure $4\left(\right.$ b) shows $F_{\mathrm{G}}$ as a function of the channel repeated times $n$ for six different arbitrary channels. For $n=1$, the evaluated average fidelity for the six arbitrary channels is $97 \%$. Figures $4(\mathrm{~d})$ and 4(e) show the typical $\chi_{\mathrm{E}}$ and $\chi_{\mathrm{T}}$ of the 4th channel at $n=1$ and $n=10$, respectively, demonstrating excellent agreement between experiment and theory. As an alternative measure, the diamond distance $D_{\diamond}$ [3] is also applied to evaluate the performance of our experimental QCS, as depicted in Fig. 4(c). The average $D_{\diamond}$ is about 0.25 at $n=1$. It is interesting to note that the repetitive QCS with increased $n$ shows an increment of fidelity or a reduction of distance. An intuitive explanation for this behavior is that the repetitive arbitrary channel converges to a depolarization channel when both the target and experimental channels are with imperfections, and the difference between $\chi_{\mathrm{E}}$ and $\chi_{\mathrm{T}}$ reduces with increased $n$.

\section{DISCUSSION}

The demonstrated repetitive QCS is important for understanding fundamental decoherence processes in an open quantum system. It also represents a significant step towards full control of an open quantum system, which promises to manipulate and stabilize quantum states, as well as realize general positive-operator valued measure. Inspired by Lloyd and Viola [49], it was proved that QCS of arbitrary dimension can be efficiently realized with a single ancilla qubit and adaptive control [36]. Therefore, the demonstrated QCS scheme can also be generalized to a photonic qudit with $d$-levels [36], using exactly the same experimental setup and requiring only a single ancilla qubit and about $2 \log _{2} d$ steps of adaptive control. Our work thus paves the way to arbitrary QCS of a bosonic oscillator with high-dimensional Hilbert space.

It is worth noting that our ultrafast adaptive control allows for the implementation of channels within a duration of $\mu \mathrm{s}$ and thus provides a platform for simulating continuous dynamics of quantum systems in an artificial environment. It was proposed in Refs. [50, 51] that an arbitrary quantum channel can also be simulated with a given error bound by the Suzuki-LieTrotter decomposition, similar to the arbitrary Hamiltonian construction [52]. Therefore, instead of a convex combination of quasiextreme channels as demonstrated, our experimental architecture with ultrafast adaptive control is also suitable for implementing an arbitrary Liouvillian $\mathscr{L}=\sum_{j} \mathscr{L}_{j}$ by alternatively and piecewisely simulating its components $\mathscr{L}_{j}$ [Supplementary Information]. In addition, although our experimental results show high fidelity of state generation by the simulated quantum channel, further improvement calls for incorporating quantum error correction $[31,32]$ into this scheme.

\footnotetext{
* These two authors contributed equally to this work.

$\dagger$ clzou321@ustc.edu.cn
} 
$\ddagger$ luyansun@tsinghua.edu.cn

[1] M. A. Nielsen and I. L. Chuang, Quantum Computation and Quantum Information (Cambridge Univ. Press, 2000).

[2] F. Petruccione and H. Breuer, The theory of open quantum systems (Oxford University Press, 2002) p. 625.

[3] M. M. Wilde, Quantum Information Theory (Cambridge University Press, 2013).

[4] E. Knill and R. Laflamme, "Power of one bit of quantum information,” Phys. Rev. Lett. 81, 5672 (1998).

[5] W. Wang, B. Yadin, Y. Ma, J. Ma, Y. Xu, L. Hu, H. Wang, Y. P. Song, M. Gu, and L. Sun, "Witnessing quantum resource conversion within deterministic quantum computation using one pure superconducting qubit," arXiv:1806.05543 (2018).

[6] F. Verstraete, M. M. Wolf, and J. Ignacio Cirac, "Quantum computation and quantum-state engineering driven by dissipation," Nat. Phys. 5, 633 (2009).

[7] S. Shankar, M. Hatridge, Z. Leghtas, K. M. Sliwa, a. Narla, U. Vool, S. M. Girvin, L. Frunzio, M. Mirrahimi, and M. H. Devoret, "Autonomously stabilized entanglement between two superconducting quantum bits." Nature 504, 419 (2013).

[8] F. Reiter, A. S. Sørensen, P. Zoller, and C. A. Muschik, "Dissipative quantum error correction and application to quantum sensing with trapped ions," Nat. Commun. 8, 1822 (2017).

[9] S. Touzard, A. Grimm, Z. Leghtas, S. O. Mundhada, P. Reinhold, C. Axline, M. Reagor, K. Chou, J. Blumoff, K. M. Sliwa, S. Shankar, L. Frunzio, R. J. Schoelkopf, M. Mirrahimi, and M. H. Devoret, "Coherent Oscillations inside a Quantum Manifold Stabilized by Dissipation,” Phys. Rev. X 8, 021005 (2018).

[10] J. Eisert, M. Friesdorf, and C. Gogolin, "Quantum many-body systems out of equilibrium,” Nat. Phys. 11, 124 (2015).

[11] C. Neill, P. Roushan, M. Fang, Y. Chen, M. Kolodrubetz, Z. Chen, A. Megrant, R. Barends, B. Campbell, B. Chiaro, A. Dunsworth, E. Jeffrey, J. Kelly, J. Mutus, P. J. J. O’Malley, C. Quintana, D. Sank, A. Vainsencher, J. Wenner, T. C. White, A. Polkovnikov, and J. M. Martinis, "Ergodic dynamics and thermalization in an isolated quantum system," Nat. Phys. 12, 1037 (2016).

[12] S. Diehl, E. Rico, M. a. Baranov, and P. Zoller, "Topology by dissipation in atomic quantum wires," Nat. Phys. 7, 971 (2011).

[13] C. Gardiner, P. Zoller, and P. Zoller, Quantum noise: a handbook of Markovian and non-Markovian quantum stochastic methods with applications to quantum optics, Vol. 56 (Springer Science \& Business Media, 2004).

[14] V. V. Albert, B. Bradlyn, M. Fraas, and L. Jiang, "Geometry and Response of Lindbladians," Phys. Rev. X 6, 041031 (2016).

[15] P. Zanardi, J. Marshall, and L. Campos Venuti, "Dissipative universal Lindbladian simulation,” Phys. Rev. A 93, 022312 (2016).

[16] A. Bienfait, J. J. Pla, Y. Kubo, X. Zhou, M. Stern, C. C. Lo, C. D. Weis, T. Schenkel, D. Vion, D. Esteve, J. J. L. Morton, and P. Bertet, "Controlling spin relaxation with a cavity," Nature 531, 74 (2016).

[17] J. T. Barreiro, M. Müller, P. Schindler, D. Nigg, T. Monz, M. Chwalla, M. Hennrich, C. F. Roos, P. Zoller, and R. Blatt, "An open-system quantum simulator with trapped ions." Nature 470, 486 (2011).

[18] P. Schindler, M. Müller, D. Nigg, J. T. Barreiro, E. A. Martinez, M. Hennrich, T. Monz, S. Diehl, P. Zoller, and R. Blatt, "Quantum simulation of dynamical maps with trapped ions," Nat. Phys. 9, 361 (2013).

[19] B. M. Terhal, I. L. Chuang, D. P. DiVincenzo, M. Grassl, and J. A. Smolin, "Simulating quantum operations with mixed environments," Phys. Rev. A 60, 881 (1999).

[20] D. S. Wang, D. W. Berry, M. C. De Oliveira, and B. C. Sanders,
"Solovay-kitaev decomposition strategy for single-qubit channels," Phys. Rev. Lett. 111, 130504 (2013).

[21] H. Lu, C. Liu, D.-S. Wang, L.-K. Chen, Z.-D. Li, X.-C. Yao, L. Li, N.-L. Liu, C.-Z. Peng, B. C. Sanders, Y.-A. Chen, and J.-W. Pan, "Experimental quantum channel simulation," Phys. Rev. A 95, 042310 (2017).

[22] W. McCutcheon, A. McMillan, J. G. Rarity, and M. S. Tame, "Experimental demonstration of a measurement-based realisation of a quantum channel," New J. Phys. 20, 033019 (2018).

[23] A. Wallraff, D. I. Schuster, A. Blais, L. Frunzio, R.-S. Huang, J. Majer, S. Kumar, S. M. Girvin, and R. J. Schoelkopf, "Circuit quantum electrodynamics: Coherent coupling of a single photon to a cooper pair box," Nature 431, 162 (2004).

[24] H. Paik, D. I. Schuster, L. S. Bishop, G. Kirchmair, G. Catelani, A. P. Sears, B. R. Johnson, M. J. Reagor, L. Frunzio, L. I. Glazman, S. M. Girvin, M. H. Devoret, and R. J. Schoelkopf, "Observation of High Coherence in Josephson Junction Qubits Measured in a Three-Dimensional Circuit QED Architecture," Phys. Rev. Lett. 107, 240501 (2011).

[25] M. H. Devoret and R. J. Schoelkopf, "Superconducting circuits for quantum information: an outlook." Science 339, 1169 (2013).

[26] Q. Ficheux, S. Jezouin, Z. Leghtas, and B. Huard, "Dynamics of a qubit while simultaneously monitoring its relaxation and dephasing," Nat. Commun. 9, 1926 (2018).

[27] S. Vinjanampathy and J. Anders, "Quantum thermodynamics," Contemp. Phys. 57, 545 (2016).

[28] R. Alicki and R. Kosloff, "Introduction to quantum thermodynamics: History and prospects," arXiv:1801.08314 (2018).

[29] R. Laurenza, C. Lupo, G. Spedalieri, S. L. Braunstein, and S. Pirandola, "Channel Simulation in Quantum Metrology," Quantum Meas. Quantum Metrol. 5, 1 (2018).

[30] H. Yuan and C.-H. F. Fung, "Fidelity and Fisher information on quantum channels," New J. Phys. 19, 113039 (2017).

[31] N. Ofek, A. Petrenko, R. Heeres, P. Reinhold, Z. Leghtas, B. Vlastakis, Y. Liu, L. Frunzio, S. M. Girvin, L. Jiang, M. Mirrahimi, M. H. Devoret, and R. J. Schoelkopf, "Extending the lifetime of a quantum bit with error correction in superconducting circuits," Nature 536, 441 (2016).

[32] L. Hu, Y. Ma, W. Cai, X. Mu, Y. Xu, W. Wang, Y. Wu, H. Wang, Y. Song, C. Zou, S. M. Girvin, L.-M. Duan, and L. Sun, "Demonstration of quantum error correction and universal gate set on a binomial bosonic logical qubit," arXiv:1805.09072 (2018).

[33] A. A. Houck, H. E. Türeci, and J. Koch, "On-chip quantum simulation with superconducting circuits," Nat. Phys. 8, 292 (2012).

[34] Y. Salathé, M. Mondal, M. Oppliger, J. Heinsoo, P. Kurpiers, A. Potočnik, A. Mezzacapo, U. Las Heras, L. Lamata, E. Solano, S. Filipp, and A. Wallraff, "Digital quantum simulation of spin models with circuit quantum electrodynamics," Phys. Rev. X 5, 021027 (2015).

[35] E. Andersson, J. D. Cresser, and M. J. W. Hall, "Finding the Kraus decomposition from a master equation and vice versa,' J. Mod. Opt. 54, 1695 (2007).

[36] C. Shen, K. Noh, V. V. Albert, S. Krastanov, M. H. Devoret, R. J. Schoelkopf, S. M. Girvin, and L. Jiang, "Quantum channel construction with circuit quantum electrodynamics," Phys. Rev. B , 134501 (2017).

[37] G. Kirchmair, B. Vlastakis, Z. Leghtas, S. E. Nigg, H. Paik, E. Ginossar, M. Mirrahimi, L. Frunzio, S. M. Girvin, and R. J. Schoelkopf, "Observation of quantum state collapse and revival due to the single-photon Kerr effect," Nature 495, 205 (2013).

[38] B. Vlastakis, G. Kirchmair, Z. Leghtas, S. E. Nigg, L. Frun- 
zio, S. M. Girvin, M. Mirrahimi, M. H. Devoret, and R. J. Schoelkopf, "Deterministically encoding quantum information using 100-photon Schrödinger cat states." Science 342, 607 (2013).

[39] L. Sun, A. Petrenko, Z. Leghtas, B. Vlastakis, G. Kirchmair, K. M. Sliwa, A. Narla, M. Hatridge, S. Shankar, J. Blumoff, L. Frunzio, M. Mirrahimi, M. H. Devoret, and R. J. Schoelkopf, "Tracking photon jumps with repeated quantum non-demolition parity measurements," Nature 511, 444 (2014).

[40] K. Liu, Y. Xu, W. Wang, S.-B. Zheng, T. Roy, S. Kundu, M. Chand, A. Ranadive, R. Vijay, Y. Song, L. Duan, and L. Sun, "A twofold quantum delayed-choice experiment in a superconducting circuit," Sci. Adv. 3, e1603159 (2017).

[41] M. Hatridge, R. Vijay, D. H. Slichter, J. Clarke, and I. Siddiqi, "Dispersive magnetometry with a quantum limited SQUID parametric amplifier," Phys. Rev. B 83, 134501 (2011).

[42] T. Roy, S. Kundu, M. Chand, A. M. Vadiraj, A. Ranadive, N. Nehra, M. P. Patankar, J. Aumentado, A. A. Clerk, and R. Vijay, "Broadband parametric amplification with impedance engineering: Beyond the gain-bandwidth product," Appl. Phys. Lett. 107, 262601 (2015).

[43] A. Kamal, A. Marblestone, and M. H. Devoret, "Signal-topump back action and self-oscillation in double-pump Josephson parametric amplifier,” Phys. Rev. B 79, 184301 (2009).

[44] K. W. Murch, S. J. Weber, C. Macklin, and I. Siddiqi, “Observing single quantum trajectories of a superconducting quantum bit," Nature 502, 211 (2013).

[45] N. Khaneja, T. Reiss, C. Kehlet, T. Schulte-Herbrüggen, and S. J. Glaser, "Optimal control of coupled spin dynamics: design of nmr pulse sequences by gradient ascent algorithms," J. Magn. Reson. 172, 296 (2005).

[46] P. De Fouquieres, S. Schirmer, S. Glaser, and I. Kuprov, "Second order gradient ascent pulse engineering," J. Magn. Reson. 212, 412 (2011).

[47] J. R. Johansson, P. D. Nation, and F. Nori, "Qutip: An opensource python framework for the dynamics of open quantum systems," Comp. Phys. Comm. 183, 1760 (2012).

[48] J. R. Johansson, P. D. Nation, and F. Nori, "Qutip 2: A python framework for the dynamics of open quantum systems," Comp. Phys. Comm. 184, 1234 (2013).

[49] S. Lloyd and L. Viola, "Engineering quantum dynamics," Phys. Rev. A 65, 010101 (2001).

[50] M. Kliesch, T. Barthel, C. Gogolin, M. Kastoryano, and J. Eisert, "Dissipative Quantum Church-Turing Theorem," Phys. Rev. Lett. 107, 120501 (2011).

[51] R. Sweke, I. Sinayskiy, and F. Petruccione, "Simulation of single-qubit open quantum systems," Phys. Rev. A 90, 022331 (2014).

[52] S. Lloyd, “Universal Quantum Simulators," Science 273, 1073 (1996).

\section{Acknowledgments}

We thank N. Ofek and Y. Liu for valuable suggestions on FPGA programming. CLZ is supported by the National Key Research and Development Program of China (Grant No.2016YFA0301300, 2017YFA0304504) and Anhui Initiative in Quantum Information Technologies (AHY130000). LS acknowledges the support from National Natural Science Foundation of China Grant No.11474177, National Key Research and Development Program of China No.2017YFA0304303, and the Thousand Youth Fellowship program in China. LS also thanks R. Vijay and his group for help on the parametric amplifier measurements. 


\title{
Supplementary Information "Experimental repetitive quantum channel simulation"
}

\author{
L. Hu, ${ }^{1, *}$ X. Mu, ${ }^{1, *}$ W. Cai, ${ }^{1}$ Y. Ma,${ }^{1}$ Y. Xu, ${ }^{1}$ H. Wang, ${ }^{1}$ Y. P. Song, ${ }^{2}$ C.-L. Zou, ${ }^{2, \dagger}$ and L. Sun ${ }^{1, *}$ \\ ${ }^{1}$ Center for Quantum Information, Institute for Interdisciplinary Information Sciences, Tsinghua University, Beijing 100084, China \\ ${ }^{2}$ Key Laboratory of Quantum Information, CAS, University of Science and Technology of China, Hefei, Anhui 230026, P. R. China
}




\section{EXPERIMENTAL SYSTEM AND SETUP}

Our experimental device is measured in a dilution refrigerator with a base temperature of about $10 \mathrm{mK}$. The device consists of an ancillary transmon qubit dispersively coupled to both a readout cavity and a storage cavity. The ancilla qubit is fabricated on a $c$-plane sapphire $\left(\mathrm{Al}_{2} \mathrm{O}_{3}\right)$ substrate with the standard double-angle evaporation of aluminum and electron-beam lithography. The rectangular cavities are made of high purity $5 \mathrm{~N} 5$ aluminum, chemically etched for a better coherence time $[1,2]$. The ancillary transmon qubit has a frequency $\omega_{\mathrm{q}} / 2 \pi=5.692 \mathrm{GHz}$, an energy relaxation time $T_{1}=30 \mu \mathrm{s}$, and a pure phasing time $T_{\phi}=120 \mu \mathrm{s}$. The storage cavity has a frequency $\omega_{\mathrm{s}} / 2 \pi=7.634 \mathrm{GHz}$, a single-photon lifetime $T_{1}^{\mathrm{s}}=143 \mu \mathrm{s}$, and a coherence time $T_{2}^{\mathrm{s}}=250 \mu \mathrm{s}$. Fock states $|0\rangle$ and $|1\rangle$ of the storage cavity constitute the two bases of a photonic qubit on which channel simulations are realized. The dephasing, damping, and arbitrary channels all rely on the dispersive interaction $\chi_{\mathrm{s}} / 2 \pi=1.90 \mathrm{MHz}$ between the ancilla and the photonic qubit. The readout cavity is at a frequency of $\omega_{\mathrm{r}} / 2 \pi=8.610 \mathrm{GHz}$, and has a lifetime of $44 \mathrm{~ns}$ and a dispersive interaction $\chi_{\mathrm{r}} / 2 \pi=3.65 \mathrm{MHz}$ with the ancilla. With the help of a Josephson parametric Amplifier (JPA) [3-6] as the first stage of amplification, quantum non-demolition single-shot measurements of the ancilla qubit can be realized with high fidelities: $>99.9 \%$ for the ground state $|g\rangle$ and $98.9 \%$ for the excited state $|e\rangle$ in a duration of 320 ns. Therefore, each readout measurement throughout our experiment returns a digitized value of the ancilla qubit state.

Fast real-time adaptive control is vital for the demonstrated channel simulations. This is achieved through three field programmable gate arrays (FPGA) with home-made logics, which are able to integrate readout signal sampling, ancilla state estimation, and manipulation signal generation together, and also allow us to individually control the ancilla qubit, the photonic qubit, and the readout cavity. The latency time, defined as the time interval between sending out the last point of the readout signal and sending out the first point of the control signal, is $340 \mathrm{~ns}$ (about $1 \%$ of the ancilla qubit lifetime). This time includes the signal travel time through the whole experimental circuitry. More details of the experimental setup and FPGAs can be found in our earlier report in Ref. 7.

\section{GENERAL THEORY}

\section{A. Quantum channel and process matrix}

The Kraus representation of a quantum channel or quantum operation can be written as [8]

$$
\rho \mapsto \mathscr{E}(\rho)=\sum_{k=1}^{M} E_{k} \rho E_{k}^{\dagger},
$$

with $1 \leq M \leq d^{2}$ ( $d$ is the Hilbert space dimension) and the Kraus operators satisfy

$$
\sum_{k} E_{k}^{\dagger} E_{k}=\mathbf{I}
$$

where $\mathbf{I}$ is the unity matrix.

Representing the Kraus operators in a certain basis, the channel can also be expressed based on the process $\chi$ matrix [9]

$$
\rho \mapsto \sum_{m, n} \chi_{m n} \widetilde{E_{m}} \rho{\widetilde{E_{n}}}^{\dagger},
$$

where the $\chi$ matrix contains $d^{4}-d^{2}$ independent parameters under the constraints that

$$
\sum_{m, n} \chi_{m n} \widetilde{E_{n}}{ }^{\dagger} \widetilde{E_{m}}=\mathbf{I} \text {. }
$$

Usually, we choose Pauli matrices as the operation elements, with the definition consistent with those in [8]:

$$
\widetilde{E_{m}} \in\{\mathbf{I}, \mathbf{X},-i \mathbf{Y}, \mathbf{Z}\}
$$

with

$$
\mathbf{X}=\left[\begin{array}{ll}
0 & 1 \\
1 & 0
\end{array}\right], \mathbf{Y}=\left[\begin{array}{cc}
0 & -i \\
i & 0
\end{array}\right], \mathbf{Z}=\left[\begin{array}{cc}
1 & 0 \\
0 & -1
\end{array}\right]
$$

and the quantum state $\alpha|0\rangle+\beta|1\rangle$ is written in a vector notation as

$$
\left[\begin{array}{l}
\alpha \\
\beta
\end{array}\right]
$$

In the following, we provide details of the basic channels studied in this work. 


\section{Identity channel}

The identity channel is trivial:

$$
\mathscr{E}(\rho)=\rho
$$

and the corresponding process matrix is

$$
\chi_{\mathrm{I}}=\left[\begin{array}{llll}
1 & 0 & 0 & 0 \\
0 & 0 & 0 & 0 \\
0 & 0 & 0 & 0 \\
0 & 0 & 0 & 0
\end{array}\right]
$$

To characterize the ability of quantum channel $\chi$ for keeping quantum information, we introduce the process fidelity as

$$
F_{\chi}=\operatorname{Tr}(\chi \mathrm{I} \chi)
$$

\section{Depolarization channel}

The depolarization channel is defined as [8]

$$
\mathscr{E}(\rho)=(1-p) \mathbf{I} \rho \mathbf{I}+\frac{p}{3}(\mathbf{X} \rho \mathbf{X}+\mathbf{Y} \rho \mathbf{Y}+\mathbf{Z} \rho \mathbf{Z})
$$

and the corresponding process matrix is

$$
\chi_{\mathrm{dpl}}=\left[\begin{array}{cccc}
1-p & 0 & 0 & 0 \\
0 & \frac{p}{3} & 0 & 0 \\
0 & 0 & \frac{p}{3} & 0 \\
0 & 0 & 0 & \frac{p}{3}
\end{array}\right]
$$

The effect of this channel is to mix the input state with the completely mixed state $\mathbf{I} / 2$.

\section{Qubit dephasing channel}

The dephasing channel is defined as [8]

$$
\mathscr{E}(\rho)=E_{0} \rho E_{0}^{\dagger}+E_{1} \rho E_{1}^{\dagger}
$$

with

$$
E_{0}=\sqrt{p} \mathbf{I}, E_{1}=\sqrt{1-p} \mathbf{Z},
$$

where $p \in[0,1]$. According to Eq. (S.3), we can get the $\chi$ matrix for a dephasing channel as

$$
\chi_{\mathrm{dph}}=\left[\begin{array}{cccc}
p & 0 & 0 & 0 \\
0 & 0 & 0 & 0 \\
0 & 0 & 0 & 0 \\
0 & 0 & 0 & 1-p
\end{array}\right]
$$

After repetitively implementing the channel for $n$ times, we have the process matrix for $\mathscr{E} n$

$$
\chi_{\mathrm{dph}}^{(n)}=\left[\begin{array}{cccc}
2^{n-1}\left(p-\frac{1}{2}\right)^{n}+\frac{1}{2} & 0 & 0 & 0 \\
0 & 0 & 0 & 0 \\
0 & 0 & 0 & 0 \\
0 & 0 & 0 & \frac{1}{2}-2^{n-1}\left(p-\frac{1}{2}\right)^{n}
\end{array}\right] .
$$

Therefore, the process fidelity decays exponentially with $n$ as

$$
F_{\chi}=\frac{1}{2}\left[1+(2 p-1)^{n}\right],
$$

which converges to $F_{\chi}=\frac{1}{2}$ for $n \gg 1$. 


\section{Qubit damping channel}

The amplitude damping channel is defined as [8]

$$
\mathscr{E}(\rho)=E_{0} \rho E_{0}^{\dagger}+E_{1} \rho E_{1}^{\dagger}
$$

with

$$
E_{0}=\left[\begin{array}{cc}
1 & 0 \\
0 & \sqrt{1-\gamma}
\end{array}\right], E_{1}=\left[\begin{array}{cc}
0 & \sqrt{\gamma} \\
0 & 0
\end{array}\right],
$$

where $\gamma \in[0,1]$. According to Eq. (S.3), we can get the $\chi$ matrix for a damping channel

$$
\chi_{\mathrm{dmp}}=\left[\begin{array}{cccc}
\frac{\sqrt{1-\gamma}}{2}-\frac{\gamma}{4}+\frac{1}{2} & 0 & 0 & \frac{\gamma}{4} \\
0 & \frac{\gamma}{4} & -\frac{\gamma}{4} & 0 \\
0 & -\frac{\gamma}{4} & \frac{\gamma}{4} & 0 \\
\frac{\gamma}{4} & 0 & 0 & \frac{1}{2}-\frac{\sqrt{1-\gamma}}{2}-\frac{\gamma}{4}
\end{array}\right] .
$$

After repetitively implementing the channel for $n$ times, we have the process matrix for $\mathscr{E} n$

$$
\chi_{\mathrm{dmp}}^{(n)}=\left[\begin{array}{cccc}
\frac{\left[1+(1-\gamma)^{\frac{n}{2}}\right]^{2}}{4} & 0 & 0 & \frac{\left[1-(1-\gamma)^{n}\right]}{4} \\
0 & \frac{\left[1-(1-\gamma)^{n}\right]}{4} & -\frac{\left[1-(1-\gamma)^{n}\right]}{4} & 0 \\
0 & -\frac{\left[1-(1-\gamma)^{n}\right]}{4} & \frac{\left[1-(1-\gamma)^{n}\right]}{4} & 0 \\
\frac{\left[1-(1-\gamma)^{n}\right]}{4} & 0 & 0 & \frac{\left[1-(1-\gamma)^{\frac{n}{2}}\right]^{2}}{4}
\end{array}\right]
$$

Therefore, the process fidelity decays bi-exponentially with $n$ as

$$
F_{\chi}=\frac{1}{4}+\frac{1}{2}(1-\gamma)^{\frac{n}{2}}+\frac{1}{4}(1-\gamma)^{n},
$$

which converges to $F_{\chi}=\frac{1}{4}$ for $n \gg 1$.

\section{B. Correspondence between quantum channel simulation and master equation}

\section{Liouvillian and repetitive quantum channel simulation}

Here, we explain the correspondence between digital quantum channel simulation and master equation for continuous open system dynamics. We start the discussion with a simple example, in which the system couples to a Markovian environment through the operator $o$ as

$$
H=H_{0}+\sum_{\omega} \omega b_{\omega}^{\dagger} b_{\omega}+\sum_{\omega}\left(g_{\omega} o^{\dagger} b_{\omega}+h . c .\right)
$$

where the bosonic operator $b_{\omega}$ denotes the harmonic oscillator mode in the bath with frequency of $\omega$, and $g_{\omega}$ is the corresponding coupling strength. Applying the Born approximation, we obtain the evolution of the system density matrix as [10]

$$
\begin{aligned}
\frac{d}{d t} \rho_{\mathrm{s}}= & -i\left[H_{0}, \rho_{\mathrm{s}}\right] \\
& -\left\{\int_{0}^{t} d \tau\left[o o^{\dagger} \rho_{\mathrm{s}}(t-\tau)-o^{\dagger} \rho_{\mathrm{s}}(t-\tau) o\right] \sum_{\omega}\left|g_{\omega}^{2}\right|\left\langle b_{\omega}^{\dagger}(t) b_{\omega}(t-\tau)\right\rangle+\text { h.c. }\right\} \\
& -\left\{\int_{0}^{t} d \tau\left[o^{\dagger} o \rho_{\mathrm{s}}(t-\tau)-o \rho_{\mathrm{s}}(t-\tau) o^{\dagger}\right] \sum_{\omega}\left|g_{\omega}^{2}\right|\left\langle b_{\omega}(t) b_{\omega}^{\dagger}(t-\tau)\right\rangle+\text { h.c. }\right\} .
\end{aligned}
$$

For a low-temperature reservoir, $\left\langle b_{\omega}^{\dagger}(t) b_{\omega}(t-\tau)\right\rangle \approx 0$ and $\left\langle b_{\omega}(t) b_{\omega}^{\dagger}(t-\tau)\right\rangle=\delta(\tau)$. If we define $\kappa=\frac{1}{2} \int d \omega \xi(\omega)\left|g_{\omega}^{2}\right|$ with $\xi(\omega)$ being the density of states in the bath, we obtain the master equation in the Lindblad form as [11, 12]

$$
\begin{aligned}
\frac{d}{d t} \rho & =-i\left[H_{0}, \rho\right]+\kappa\left(2 o \rho o^{\dagger}-o^{\dagger} o \rho-\rho o^{\dagger} o\right) \\
& =\mathscr{L}_{0}(\rho)+\mathscr{L}_{1}(\rho)
\end{aligned}
$$


with Liouvillian $\mathscr{L}_{0}$ and $\mathscr{L}_{1}$ describing the coherent and incoherent evolutions, respectively. For $d t \rightarrow 0$, we have

$$
\rho(t+d t)-\rho(t)=\mathscr{L}_{0}(\rho) d t+\mathscr{L}_{1}(\rho) d t+\mathscr{O}\left[\left(H_{0} d t\right)^{2} \rho\right]+\mathscr{O}\left[(\kappa d t)^{2} \rho\right] .
$$

Through channel simulation, we can implement unitary channel

$$
\begin{aligned}
\mathscr{E}_{0}(\rho) & =e^{-i H d t} \rho e^{i H d t} \\
& =\rho-i\left[H_{0} d t, \rho\right]+\mathscr{O}\left[\left(H_{0} d t\right)^{2} \rho\right] \\
& =\rho+\mathscr{L}_{0}(\rho) d t+\mathscr{O}\left[\left(H_{0} d t\right)^{2} \rho\right]
\end{aligned}
$$

through arbitrary unitary control [13], and non-unitary channel

$$
\begin{aligned}
\mathscr{E}_{1}(\rho) & =E_{1,0} \rho E_{1,0}^{\dagger}+E_{1,1} \rho E_{1,1}^{\dagger} \\
& =\rho+\kappa d t\left(2 o \rho o^{\dagger}-o^{\dagger} o \rho-\rho o^{\dagger} o\right)+\mathscr{O}\left[(\kappa d t)^{2} \rho\right] \\
& =\mathscr{L}_{1}(\rho) d t+\mathscr{O}\left[(\kappa d t)^{2} \rho\right]
\end{aligned}
$$

with

$$
\begin{aligned}
& E_{1,0}=\sqrt{2 \kappa d t} o, \\
& E_{1,1}=\mathbf{I}-\kappa d t o^{\dagger} o .
\end{aligned}
$$

Therefore, by sequentially implementing these two channels

$$
\begin{aligned}
\mathscr{E}_{1} \circ \mathscr{E}_{0}(\rho) & =\mathscr{L}_{0}(\rho) d t+\mathscr{L}_{1}(\rho) d t+\mathscr{O}\left[\left(H_{0} d t\right)^{2} \rho\right]+\mathscr{O}\left[(\kappa d t)^{2} \rho\right] \\
& \approx e^{d t\left(\mathscr{L}_{0}+\mathscr{L}_{1}\right)} \rho,
\end{aligned}
$$

we obtain the effective implementation of the Liouvillian with an error to the order of $\left(H_{0} d t\right)^{2}+(\kappa d t)^{2}$.

The above derivations can be easily generalized to multiple-Liouvillian case, i.e. $\Sigma_{j} \mathscr{L}_{j}$. As proved in Refs. [14, 15], the effective continuous open quantum system dynamics can be realized by repetitively implementing the quantum channels which correspond to each individual Liouvillian alternatively.

\section{Qubit dephasing channel}

When $o=\mathbf{Z}$ and $H=0$ in Eq.(S.25), the master equation corresponds to a simple Markovian dephasing channel on a qubit. According to the analysis above, when

$$
\begin{aligned}
& E_{1,0}=\sqrt{2 \kappa d t} \mathbf{Z}, \\
& E_{1,1}=\sqrt{1-2 \kappa d t} \mathbf{I},
\end{aligned}
$$

the repetitive implementation of the channel (in the limit of $\kappa d t \ll 1$ ) will give us an effective continuous dephasing process. According to the definition of a dephasing channel (Eq. (S.13)), it requires that $p=1-2 \kappa d t$. It also indicates that the implementation of the digital dephasing channel simulation with $1-p \ll 1$ and a duration of $\tau_{0}$ leads to an effective continuous dephasing Liouvillian with a dephasing rate $\kappa=(1-p) / 2 \tau_{0}$, and the corresponding phase coherence time is $T_{2}=1 / 4 \kappa$.

\section{Qubit damping channel}

Similarly, when

$$
o=\sigma_{-}=\left[\begin{array}{ll}
0 & 1 \\
0 & 0
\end{array}\right]
$$


and $H=0$ in Eq. (S.25), the master equation corresponds to a simple Markovian excited state damping channel on a qubit. If

$$
\begin{aligned}
& E_{1,0}=\sqrt{2 \kappa d t} \sigma_{-}, \\
& E_{1,1}=\mathbf{I}-\kappa d t \frac{\mathbf{I}-\mathbf{Z}}{2},
\end{aligned}
$$

the repetitive implementation of the channel (in the limit of $\kappa d t \ll 1$ ) will give us an effective continuous damping process. According to the definition of a damping channel Eq. (S.19), it requires that $\gamma=2 \kappa d t$. It also indicates that the implementation of the digital damping channel simulation with $\gamma \ll 1$ and a duration of $\tau_{0}$ leads to an effective continuous damping Liouvillian with a damping rate $\kappa=\gamma / 2 \tau_{0}$.

\section{EXPERIMENTAL QUANTUM CHANNEL SIMULATIONS}

\section{A. The dephasing channel simulation}

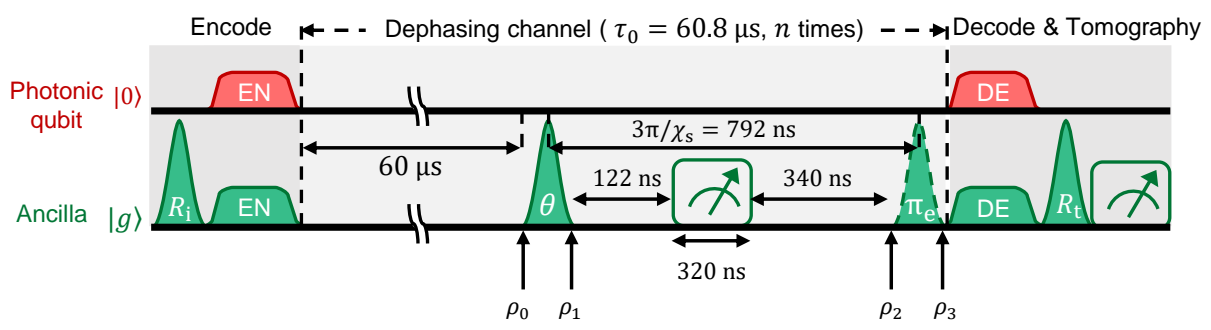

FIG. S1. Experimental sequence for a dephasing channel simulation. Density matrices at the arrows are shown in detail in the text.

In our experiment, the dephasing channel is realized by using the control pulse sequence in Fig.2(a) in the main text. To explain the protocol, we analyze the procedures in detail as follows.

1. The ancilla qubit is initialized to the ground state $\rho_{\mathrm{a}}=|g\rangle\langle g|$, and the photonic qubit is encoded in $\rho_{\mathrm{in}}$. Then the input of the system is

$$
\rho_{0}=\rho_{\mathrm{a}} \otimes \rho_{\mathrm{in}}=\left[\begin{array}{ll}
1 & 0 \\
0 & 0
\end{array}\right] \otimes\left[\begin{array}{ll}
a & b \\
c & d
\end{array}\right]
$$

2. After applying $X_{\theta}$ (a unitary operation $U_{X}(\theta)=e^{-i \frac{\theta}{2} \mathbf{x}}$ ) to the ancilla qubit, we have

$$
\begin{aligned}
\rho_{0} \mapsto \rho_{1} & =U_{\mathbf{X}}(\theta) \rho_{0} U_{\mathbf{X}}^{\dagger}(\theta) \\
& =\left[\begin{array}{cc}
\cos ^{2}\left(\frac{\theta}{2}\right) & i \sin \left(\frac{\theta}{2}\right) \cos \left(\frac{\theta}{2}\right) \\
-i \sin \left(\frac{\theta}{2}\right) \cos \left(\frac{\theta}{2}\right) & \sin ^{2}\left(\frac{\theta}{2}\right)
\end{array}\right] \otimes\left[\begin{array}{ll}
a & b \\
c & d
\end{array}\right] .
\end{aligned}
$$

3. According to the Hamiltonian $H=\omega_{s} a^{\dagger} a+\omega_{a}|e\rangle\left\langle e\left|-\chi_{s} a^{\dagger} a\right| e\right\rangle\langle e|$, the $|e\rangle|1\rangle$ state gains an extra phase $e^{i \chi_{s} t}$ for a duration of $t$. Thus, waiting for a duration of $t=3 \pi / \chi_{\mathrm{s}}$, a controlled-Z (CZ) gate between the ancilla and the photonic qubit can be realized as

$$
\begin{aligned}
\rho_{1} \mapsto \rho_{2} & =U_{\mathrm{CZ} \rho_{1} U_{\mathrm{CZ}}^{\dagger}} \\
& =\left[\begin{array}{cc}
\cos ^{2}\left(\frac{\theta}{2}\right)\left[\begin{array}{ll}
a & b \\
c & d
\end{array}\right] & i \sin \left(\frac{\theta}{2}\right) \cos \left(\frac{\theta}{2}\right)\left[\begin{array}{cc}
a & -b \\
c & -d
\end{array}\right] \\
-i \sin \left(\frac{\theta}{2}\right) \cos \left(\frac{\theta}{2}\right)\left[\begin{array}{cc}
a & b \\
-c & -d
\end{array}\right] & \sin ^{2}\left(\frac{\theta}{2}\right)\left[\begin{array}{cc}
a & -b \\
-c & d
\end{array}\right]
\end{array}\right] .
\end{aligned}
$$


4. When tracing out the ancilla qubit state, we have the output state of the photonic qubit as

$$
\begin{aligned}
\rho_{\text {out }} & =\operatorname{Tr}_{\mathrm{a}}\left(\rho_{2}\right) \\
& =\cos ^{2}\left(\frac{\theta}{2}\right)\left[\begin{array}{ll}
a & b \\
c & d
\end{array}\right]+\sin ^{2}\left(\frac{\theta}{2}\right)\left[\begin{array}{cc}
a & -b \\
-c & d
\end{array}\right] \\
& =\left[\begin{array}{cc}
a & b \cos (\theta) \\
c \cos (\theta) & d
\end{array}\right] .
\end{aligned}
$$

5. To discard the ancilla qubit state, the ancilla is measured after the $\mathrm{CZ}$ gate and a conditional $X$ operation is implemented when the measurement outcome is $|e\rangle$. This measurement and adaptive operation on the ancilla qubit can be described as

$$
\mathscr{E}_{\mathrm{MF}}\left(\rho_{\mathrm{a}}\right): \rho_{\mathrm{a}} \mapsto|g\rangle\left\langle g\left|\rho_{\mathrm{a}}\right| g\right\rangle\langle g|+X| e\rangle\left\langle e\left|\rho_{\mathrm{a}}\right| e\right\rangle\langle e| X^{\dagger},
$$

i.e.

$$
\mathscr{E}_{\mathrm{MF}}\left(\rho_{\mathrm{a}}\right): \rho_{\mathrm{a}} \mapsto\left[\begin{array}{ll}
1 & 0 \\
0 & 0
\end{array}\right] \operatorname{Tr}_{\mathrm{a}}\left(\rho_{\mathrm{a}}\right)
$$

Thus, the system state before decoding is

$$
\begin{aligned}
\rho_{2} \mapsto \rho_{3} & =\mathscr{E}_{\mathrm{MF}}\left(\rho_{2}\right)=\left[\begin{array}{ll}
1 & 0 \\
0 & 0
\end{array}\right] \otimes \operatorname{Tr}_{\mathrm{a}}\left(\rho_{2}\right) \\
& =\left[\begin{array}{ll}
1 & 0 \\
0 & 0
\end{array}\right] \otimes\left[\begin{array}{cc}
a & b \cos (\theta) \\
c \cos (\theta) & d
\end{array}\right] .
\end{aligned}
$$

Comparing the input and output of the experimental procedure, we find the obtained channel is

$$
\mathscr{E}_{\text {anc } \otimes \text { target }}=\mathscr{I}_{\text {anc }} \otimes \mathscr{E}_{\text {target }},
$$

where $\mathscr{I}: \rho \mapsto \rho$ is the identity channel and

$$
\mathscr{E}_{\text {target }}: \rho \mapsto \cos ^{2}\left(\frac{\theta}{2}\right) \mathbf{I} \rho \mathbf{I}^{\dagger}+\sin ^{2}\left(\frac{\theta}{2}\right) \mathbf{Z} \rho \mathbf{Z}^{\dagger} .
$$

According to the definition in last section [Eq. (S.13)], the dephasing channel with $p=\cos ^{2}\left(\frac{\theta}{2}\right)$ is realized.

The effects of the repetitive dephasing channel simulation and the intrinsic decoherence can simply be added together, which leads to an effective channel coherence time

$$
\frac{1}{T_{2}^{c}}=-\frac{\ln \left(2 \cos ^{2} \frac{\theta}{2}-1\right)}{\tau_{0}}+\frac{1}{T_{2}^{0}},
$$

where $T_{2}^{0}$ is the intrinsic coherence time (when $\theta=0^{\circ}$ ) and $\tau_{0}$ is the time interval. In the main text, we get $T_{2}^{0}=240 \pm 4 \mu \mathrm{s}$, which agrees well with $T_{2}^{\mathrm{s}}=250 \mu \mathrm{s}$.

In our experiments, the duration of the digital quantum channel simulation is variable. When $\tau_{0} \ll T_{1}^{0}, T_{2}^{0}$, according to the discussions on the correspondence between continuous Liouvillian and repetitive channel simulation, the intrinsic dephasing and damping of the photonic qubit can also be treated as two channels. Therefore, the resulting effective dynamics when $\theta \ll 1$ can be approximated by a simple combination of the decoherence processes:

$$
\frac{1}{T_{2}^{c}} \approx \frac{2 \sin ^{2}\left(\frac{\theta}{2}\right)}{\tau_{0}}+\frac{1}{T_{2}^{0}} .
$$

\section{B. The damping channel simulation}

In our experiment, the damping channel is realized by using the control pulse sequence in Fig. 3(a) in the main text. To explain the protocol, we analyze the procedures in detail as follows. 


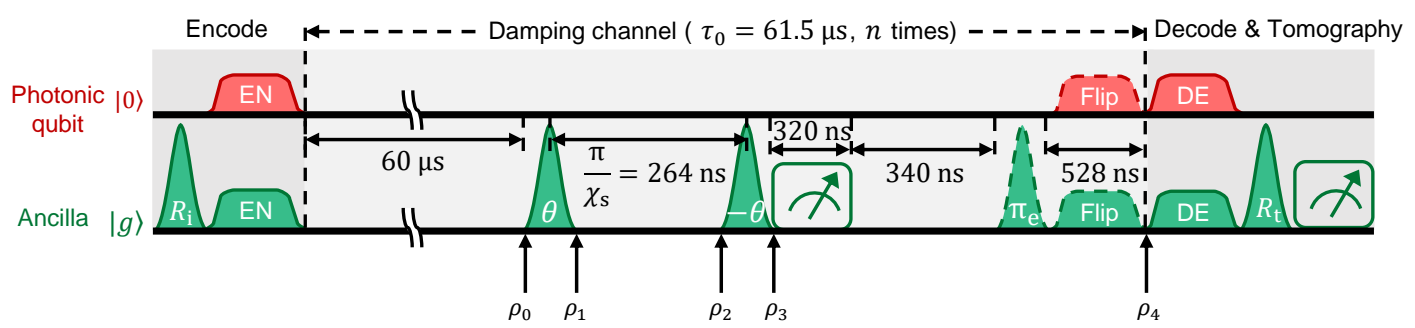

FIG. S2. Experimental sequence for a damping channel simulation. Density matrices at the arrows are shown in detail in the text.

1. The initial state of photonic qubit is $\rho_{\text {in }}$, and the ancilla qubit is initialized to ground state $\rho_{\mathrm{a}}=|g\rangle\langle g|$. So, the input system of the system is

$$
\rho_{0}=\rho_{\mathrm{a}} \otimes \rho_{\mathrm{in}}=\left[\begin{array}{ll}
1 & 0 \\
0 & 0
\end{array}\right] \otimes\left[\begin{array}{ll}
a & b \\
c & d
\end{array}\right]
$$

2. After applying $X_{\theta}\left(U_{X}(\theta)=e^{-i \frac{\theta}{2}} \mathbf{X}\right)$ to the ancilla qubit, we have

$$
\begin{aligned}
\rho_{0} \mapsto \rho_{1} & =U_{\mathbf{X}}(\theta) \rho_{0} U_{\mathbf{X}}^{\dagger}(\theta) \\
& =\left[\begin{array}{cc}
\cos ^{2}\left(\frac{\theta}{2}\right) & i \sin \left(\frac{\theta}{2}\right) \cos \left(\frac{\theta}{2}\right) \\
-i \sin \left(\frac{\theta}{2}\right) \cos \left(\frac{\theta}{2}\right) & \sin ^{2}\left(\frac{\theta}{2}\right)
\end{array}\right] \otimes\left[\begin{array}{ll}
a & b \\
c & d
\end{array}\right] .
\end{aligned}
$$

3. Due to the dispersive ancilla-photonic qubit interaction, a controlled-Z (CZ) gate can be obtained after a duration of $\pi / \chi_{\mathrm{s}}$, thus

$$
\begin{aligned}
\rho_{1} \mapsto \rho_{2} & =U_{\mathrm{CZ}} \rho_{1} U_{\mathrm{CZ}}^{\dagger} \\
& =\left[\begin{array}{cc}
\cos ^{2}\left(\frac{\theta}{2}\right)\left[\begin{array}{ll}
a & b \\
c & d
\end{array}\right] & i \sin \left(\frac{\theta}{2}\right) \cos \left(\frac{\theta}{2}\right)\left[\begin{array}{cc}
a & -b \\
c & -d
\end{array}\right] \\
-i \sin \left(\frac{\theta}{2}\right) \cos \left(\frac{\theta}{2}\right)\left[\begin{array}{cc}
a & b \\
-c & -d
\end{array}\right] & \sin ^{2}\left(\frac{\theta}{2}\right)\left[\begin{array}{cc}
a & -b \\
-c & d
\end{array}\right]
\end{array}\right] .
\end{aligned}
$$

4. After applying the second $X_{-\theta}$ (a unitary operation $U_{X}(-\theta)=e^{i \frac{\theta}{2}} \mathbf{X}$ ) to the ancilla qubit, we have

$$
\left.\begin{array}{rl}
\rho_{2} \mapsto \rho_{3} & =U_{\mathbf{X}}(-\theta) \rho_{2} U_{\mathbf{X}}^{\dagger}(-\theta) \\
& =\left[\begin{array}{cc}
a & b \cos (\theta) \\
c \cos (\theta) & d \cos ^{2}(\theta)
\end{array}\right] \quad\left[\begin{array}{cc}
0 & -i b \sin (\theta) \\
0 & -i d \cos (\theta) \sin (\theta)
\end{array}\right] \\
{\left[\begin{array}{cc}
0 & 0 \\
i c \sin (\theta) & i d \cos (\theta) \sin (\theta)
\end{array}\right]} & {\left[\begin{array}{cc}
0 \\
0 & d \sin ^{2}(\theta)
\end{array}\right]}
\end{array}\right] .
$$

5. After implementing a measurement-based adaptive operation on the photonic qubit, we have

$$
\begin{aligned}
\rho_{3} \mapsto \rho_{4} & =[|g\rangle\langle g| \otimes \mathbf{I}] \rho_{3}[|g\rangle\langle g| \otimes \mathbf{I}]^{\dagger}+[|e\rangle\langle e| \otimes \mathbf{X}] \rho_{3}[|e\rangle\langle e| \otimes \mathbf{X}]^{\dagger} \\
& =\left[\begin{array}{cc}
a & b \cos (\theta) \\
c \cos (\theta) & d \cos ^{2}(\theta)
\end{array}\right]+\left[\begin{array}{cc}
d \sin ^{2}(\theta) & 0 \\
0 & 0
\end{array}\right] \\
& =\left[\begin{array}{cc}
a+d \sin ^{2}(\theta) & b \cos (\theta) \\
c \cos (\theta) & d \cos ^{2}(\theta)
\end{array}\right] .
\end{aligned}
$$

Comparing to the definition for a damping channel (Eq.S.19), we realize a damping channel to the photonic qubit with $\gamma=$ $\sin ^{2}(\theta)$.

Similar to the case of the dephasing channel, we have

$$
\begin{gathered}
\frac{1}{T_{1}^{c}}=-\frac{\ln \left(\cos ^{2} \theta\right)}{\tau_{0}}+\frac{1}{T_{1}^{0}}, \\
\frac{1}{T_{2}^{c}}=-\frac{\ln \left(\cos ^{2} \theta\right)}{2 \tau_{0}}+\frac{1}{T_{2}^{0}},
\end{gathered}
$$


where $\tau_{0}$ is the time interval, and $T_{1}^{0}, T_{2}^{0}$ are the intrinsic decoherence times of the photonic qubit $\left(\theta=0^{\circ}\right)$. In the main text, we get $T_{1}^{0}=137 \pm 4 \mu \mathrm{s}$ and $T_{2}^{0}=237 \pm 3 \mu \mathrm{s}$, which agree well with $T_{1}^{\mathrm{s}}=143 \mu \mathrm{s}$ and $T_{2}^{\mathrm{s}}=250 \mu \mathrm{s}$, respectively.

\section{Arbitrary channel simulation}

\section{Simulation of arbitrary quantum channel}

Following the proposal by Wang et al. [16], we realize the arbitrary single-qubit channel by a convex combination of quasiextreme channels, where only a single ancilla qubit is required. As shown in Fig.1(c) in the main text, the ancilla qubit is firstly prepared to a superposition state as

$$
\rho_{\mathrm{a}}=X\left(\gamma_{p}\right)|g\rangle\langle g| X^{\dagger}\left(\gamma_{p}\right)
$$

where $X(\theta)=e^{-i \frac{\theta}{2} \mathbf{X}}=\cos \frac{\theta}{2} \mathbf{I}-i \sin \frac{\theta}{2} \mathbf{X}$. Then, a projective measurement on the ancilla qubit in the computational basis is applied, and different channel branches are implemented according to the measurement result. This can be represented by the quantum instrument [17]

$$
\mathscr{E}: \rho \mapsto \mathscr{E}_{1} \otimes|g\rangle\left\langle g\left|+\mathscr{E}_{2} \otimes\right| e\right\rangle\langle e|
$$

Here, $\mathscr{E}_{j}$ with $j=0,1$ is the quasiextreme channel, which can be represented as

$$
\begin{aligned}
\mathscr{E}_{j}: \rho \mapsto & U(\vec{\varphi})[|g\rangle\langle g| \otimes I] X\left(2 \gamma_{2}\right) U_{C Z}\left[X\left(2 \gamma_{1}\right) \otimes U(\vec{\delta})\right] \rho\left\{U(\vec{\varphi})[|g\rangle\langle g| \otimes I] X\left(2 \gamma_{2}\right) U_{C Z}\left[U(\vec{\delta}) \otimes X\left(2 \gamma_{1}\right)\right]\right\}^{\dagger} \\
& \left.+U(\vec{\varphi})[|e\rangle\langle e| \otimes X] X\left(2 \gamma_{2}\right) U_{C Z}\left[X\left(2 \gamma_{1}\right) \otimes U(\vec{\delta})\right] \rho\left\{U(\vec{\varphi})[|e\rangle\langle e| \otimes X] X\left(2 \gamma_{2}\right) U_{C Z}\left[U(\vec{\delta}) \otimes X\left(2 \gamma_{1}\right)\right]\right\}^{\dagger} \mathrm{S} .73\right)
\end{aligned}
$$

with parameters $\left\{\overrightarrow{\delta^{(j)}}, \gamma_{1}^{(j)}, \gamma_{2}^{(j)}, \overrightarrow{\varphi^{(j)}}\right\}$, and unitary operation

$$
U(\vec{\varphi})=\left[\begin{array}{cc}
\cos \frac{\varphi_{1}}{2}-i \sin \frac{\varphi_{1}}{2} \cos \frac{\varphi_{2}}{2} & -i \sin \frac{\varphi_{1}}{2} \sin \frac{\varphi_{2}}{2} e^{-i \varphi_{3}} \\
-i \sin \frac{\varphi_{1}}{2} \sin \frac{\varphi_{2}}{2} e^{i \varphi_{3}} & \cos \frac{\varphi_{1}}{2}+i \sin \frac{\varphi_{1}}{2} \cos \frac{\varphi_{2}}{2}
\end{array}\right]
$$

with $\varphi_{1,2,3} \in[0,2 \pi]$. Therefore, there are 17 parameters in total, including $\gamma_{p}$ and two sets of 8 parameters for quasiextreme channels. Actually, the dephasing and damping channels are two examples of quasiextreme channels. For a dephasing channel, we have $\gamma_{p}=0$ and $\left\{\overrightarrow{\delta^{(0)}}, \gamma_{1}^{(0)}, \gamma_{2}^{(0)}, \overrightarrow{\varphi^{(0)}}\right\}=\{(0,0,0), 0, \theta,(0,0,1)\}$. For a damping channel, we have $\gamma_{p}=0$ and $\left\{\overrightarrow{\delta^{(0)}}, \gamma_{1}^{(0)}, \gamma_{2}^{(0)}, \overrightarrow{\varphi^{(0)}}\right\}=\{(0,0,0), \theta,-\theta,(0,0,1)\}$.

\section{Random qubit channel}

In our experiment, to demonstrate the ability of simulating arbitrary single-qubit channel, we first generate a single-qubit channel numerically with 12 random numbers in a target process matrix $\chi_{\mathrm{T}}$ that satisfy certain constraints [9]. For the experimental results shown in the main text, we generated 6 random qubit channels as the targets for arbitrary quantum channel simulation. Here, we show the target $\chi_{\mathrm{T}}$ for channel arbi. 1 as an example

$$
\chi_{\mathrm{T}}^{(1)}=\left(\begin{array}{cccc}
0.362762 & 0.0143269+0.0767813 i & -0.0305766+0.075406 i & -0.00589476-0.134834 i \\
0.0143269-0.0767813 i & 0.22118 & 0.00589476+0.0979485 i & -0.0331165-0.075406 i \\
-0.0305766-0.075406 i & 0.00589476-0.0979485 i & 0.300734 & 0.0143269+0.027254 i \\
-0.00589476+0.134834 i & -0.0331165+0.075406 i & 0.0143269-0.027254 i & 0.115325
\end{array}\right)_{(S .75)}
$$

For a given target process matrix $\chi_{\mathrm{T}}$, the 17 parameters for the arbitrary channel simulation are determined through numerical optimization that reduces the difference between $\chi_{\text {sim }}$ and $\chi_{\mathrm{T}}$. Here, $\chi_{\text {sim }}$ is the simulation result for the quantum circuit shown in Fig. 1(c) in the main text. Then, we implement the channel with the experimental sequence shown in Fig. 4(a) in the main text 
with the optimized 17 parameters. The obtained experimental quantum channel $\chi_{\mathrm{E}}$ for the arbi. 1 is

$$
\chi_{\mathrm{E}}^{(1)}=\left(\begin{array}{cccc}
0.352118 & 0.0205553+0.0697286 i & -0.0117642+0.0543421 i & -0.00241937-0.107611 i \\
0.0205553-0.0697286 i & 0.244587 & 0.00241937+0.0722139 i & -0.0386366-0.0543421 i \\
-0.0117642-0.0543421 i & 0.00241937-0.0722139 i & 0.251896 & 0.0205553+0.0262903 i \\
-0.00241937+0.107611 i & -0.0386366+0.0543421 i & 0.0205553-0.0262903 i & 0.151398
\end{array}\right) .
$$

\section{Characterization of quantum channels}

To characterize the performance of the experiment for arbitrary quantum channel simulation, we introduce two different figures of merit. The first one is the fidelity of state generation

$$
F_{\mathrm{G}}=\inf _{\rho} \sqrt{\sqrt{\mathscr{E}_{\mathrm{T}}(\rho)}\left[\mathscr{E}_{\mathrm{E}}(\rho)\right] \sqrt{\mathscr{E}_{\mathrm{T}}(\rho)}} .
$$

The physical meaning of $F_{\mathrm{G}}$ is the fidelity of the output quantum state $\mathscr{E}_{\mathrm{E}}(\rho)$ when we use the quantum channel to manipulate the quantum state for a given input compared with the result of the target quantum channel $\mathscr{E}_{\mathrm{T}}(\rho)$. Therefore, $F_{\mathrm{G}}$ is the worst fidelity of the quantum state generated by the experimental channel.

The other one is the measure of discriminating quantum channels. For two channels $\mathscr{E}_{1}$ and $\mathscr{E}_{2}$, the diamond distance is [17]

$$
D_{\diamond}=\left\|\mathscr{E}_{1}-\mathscr{E}_{2}\right\|_{\diamond}=\sup _{\rho}\left\|\left(\mathscr{E}_{1} \otimes \mathrm{Id}\right)(\rho)-\left(\mathscr{E}_{2} \otimes \mathrm{Id}\right)(\rho)\right\|_{1},
$$

where the trace norm

$$
\|T\|_{1} \equiv \operatorname{Tr} \sqrt{T^{\dagger} T}
$$

The diamond distance $D_{\diamond} \in[0,2]$ is related to the minimal probability $p_{\text {dist }}=\frac{1}{2}-\frac{1}{4} D_{\diamond}$ of distinguishing two channels by allowing quantum entangled states between the channel and an ancillary space.

In the main text, both $F_{\mathrm{G}}$ and $D_{\diamond}$ are shown for different number of channel repetition $n$. It is shown that $F_{G}$ increases with $n$ while $D_{\diamond}$ decreases with $n$ for certain channels. To explain this behavior, we also present $D_{\diamond}$ between the target channel $\chi_{\mathrm{T}}$ (experimental channel $\chi_{\mathrm{E}}$ ) and the depolarization channel with $p=\frac{3}{4}$

$$
\chi_{\mathrm{dpl}}=\frac{1}{4}\left[\begin{array}{llll}
1 & 0 & 0 & 0 \\
0 & 1 & 0 & 0 \\
0 & 0 & 1 & 0 \\
0 & 0 & 0 & 1
\end{array}\right] .
$$

The results are presented in Fig. S3. These results indicate that repetitive arbitrary quantum channel eventually approaches a depolarization channel, therefore the distance between the target channel and the experimental channel may be closer after several rounds of repetition.
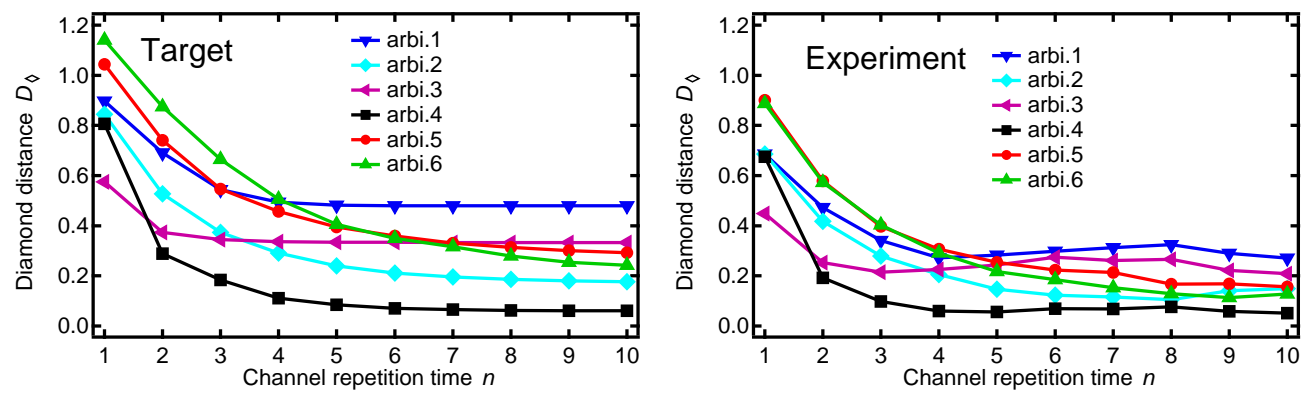

FIG. S3. Diamond distances $D_{\diamond}$ between the target channel $\chi_{\mathrm{T}}\left(\right.$ experimental channel $\left.\chi_{\mathrm{E}}\right)$ and the depolarization channel with $p=\frac{3}{4}$. 


\section{Correction of the measurement-induced phase shift}

The measurement-induced phase on the photonic qubit does not commute with other processes in the arbitrary channel simulation. So we cannot ignore it during the experiment and deal with it in the final data analysis as for the cases of the dephasing and damping channel simulations. Instead, we experimentally eliminate this extra phase by modifying the measurement-adaptive sequence, as shown in Fig. S4.

In the arbitrary channel simulation [Fig. 4(a) of the main text], there are two measurement-adaptive processes: one is to get the quantum random number for deciding different branches and the other one is used in each branch. In our experiment, we want to keep the time interval between measurement and the following rotation pulse constant ( $320 \mathrm{~ns}$ ) to minimize extra ancilla decoherence during this time interval. However, the measurement-adaptive sequence in Fig. S4(a) (used in both dephasing and damping channel simulations) will not lead to the same phase on the photonic qubit when associated with $|g\rangle$ and $|e\rangle$ respectively. To solve this problem, the measurement-adaptive sequence is replaced by Fig. S4(b). The time interval between the pre-rotation pulse on the ancilla and the measurement is increased such that the time interval between the pre-rotation and the $\pi$ pulse following the measurement is $(4 \pi-0.3) / \chi_{\mathrm{s}}$. Here $0.3 / \chi_{\mathrm{s}}$ corresponds to $17^{\circ}$ rotation of $|1\rangle$ state of the photonic qubit due to the cross Kerr between the readout cavity and the photonic qubit, independent of the ancilla state. If the measurement outcome of the ancilla state is $|e\rangle$, the $\pi$ pulse brings the ancilla to $|g\rangle$ after acquiring a total $4 \pi$ phase for $|e\rangle|1\rangle$ state (including the measurement-induced phase). Here, in the joint state notation the letters represent the ancilla states while the numbers correspond to the photonic qubit states. On the other hand, if the measurement outcome of the ancilla state is $|g\rangle$, the $\pi$ pulse after the measurement brings the ancilla to $|e\rangle$ to acquire an extra phase during the following $(2 \pi-0.3) / \chi_{\mathrm{s}}$ interval before a second conditional $\pi$ pulse flips the ancilla back to $|g\rangle$. In the end, the original $|g\rangle|1\rangle$ also acquires a $2 \pi$ phase.

(a)

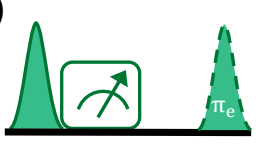

(b)

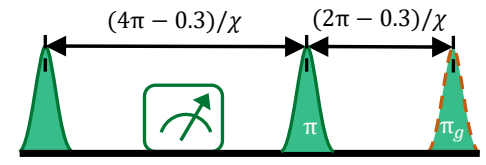

FIG. S4. Correction of the measurement-induced phase shift in the arbitrary channel simulation. The measurement-adaptive sequence (a) used in both dephasing and damping channel simulations is replaced by the new one in (b). The goal is to acquire integer multiples of $2 \pi$ phase for both $|g\rangle|1\rangle$ and $|e\rangle|1\rangle$. Here $0.3 / \chi_{\mathrm{s}}$ is the time interval for $|e\rangle|1\rangle$ state under free evolution to acquire a phase of $17^{\circ}$, which is the measurement-induced phase on both $|g\rangle|1\rangle$ and $|e\rangle|1\rangle$.

\section{DISCUSSIONS}

\section{A. The limitations of dephasing and damping rates}

The ancilla qubit facilitates all channel simulations. Consequently, its intrinsic decoherence prevents an arbitrary fast dephasing or damping rate, and eventually sets a limit on these rates. In this part, we show how the ancilla decoherence affects the simulated dephasing and damping rates in the corresponding channels.

\section{The dephasing channel}

First, we show that the dephasing of the ancilla will not affect the dephasing channel. The dephasing channel relies on the dispersive interaction between the ancilla and the photonic qubit. Explicitly, the joint state $|e\rangle|1\rangle$ acquires a phase relative to other three states $|e\rangle|0\rangle,|g\rangle|1\rangle$, and $|g\rangle|0\rangle$ with a rate equal to the dispersive interaction strength $\chi_{\mathrm{s}}$. Before the projective measurement in the channel simulation, the dephasing of the ancilla only changes the sign associated with $|e\rangle$ state, which has no observable effect on the following projective measurement and adaptive control. Therefore, the dephasing channel remains unaffected, and we only need to consider thermal excitation and ancilla decay.

After a waiting time of $60 \mu \mathrm{s}$, the ancilla qubit can be considered in a thermal equilibrium state with an $|e\rangle$ state population $n_{\mathrm{th}}=0.87 \%$ based on an independent calibration experiment. For an initial $|e\rangle$ state, the following channel simulation still works well except that the probability causing a phase flip of the photonic qubit changes from $\sin ^{2}(\theta / 2)$ to $\cos ^{2}(\theta / 2)$ due to the exchange of $|g\rangle$ and $|e\rangle$. 
Between the $X_{\theta}$ gate and the adaptive $\pi$ pulse, random decay of the ancilla qubit from $|e\rangle$ to $|g\rangle$ causes random phase on $|1\rangle$ state instead of a complete phase flip. Therefore, this will reduce the phase-flip probability of $|1\rangle$. On average, we can treat this reduction factor as $1-p_{1} / 2$ with $p_{1}=\left(3 \pi / \chi_{\mathrm{s}}\right) / T_{1}$, the probability of having an ancilla decay. The ancilla upwards transition probability from $|g\rangle$ to $|e\rangle$ is tiny during the short channel simulation process and can be neglected. Combining the above two effects, thermal excitation during $\tau_{0}$ and ancilla decay, we get the final phase flip probability for the photonic qubit as a function of the rotation angle in $X_{\theta}$ :

$$
p_{\phi}^{\lim }=\left(1-n_{\mathrm{th}}\right) \sin ^{2}(\theta / 2)\left(1-p_{1} / 2\right)+n_{\mathrm{th}} \cos ^{2}(\theta / 2)\left(1-p_{1} / 2\right) .
$$

Then we can get the upper limit of the external and controllable dephasing rate in our channel simulation:

$$
\Gamma_{\phi}^{\lim }=-\frac{\ln \left(1-2 p_{\phi}^{\lim }\right)}{\tau_{0}},
$$

which is used for Fig. 2(e) in the main text.

\section{The damping channel}

The ancilla dephasing will not affect the performance of the damping channel, provided the initial state of the photonic qubit is at $|1\rangle$ state. This is because the ancilla dephasing during the the parity-type protocol $\left(X_{\theta}, \pi / \chi_{\mathrm{s}}, X_{-\theta}\right)$ only flips the phase on $|e\rangle|1\rangle$, and this flip has no effect on the following projective measurement and adaptive control. However, if the initial state is a superposition of $\alpha|0\rangle+\beta|1\rangle$, the ancilla dephasing will affect the resulting channel. Here, we focus on the case of an initial $|1\rangle$ state of the photonic qubit, since this is a direct reflection of the damping rate of this channel [Fig. 3(b) of the main text]. Therefore, we only need to consider the thermal excitation and energy decay of the ancilla.

Similar to the case of the damping channel, after a waiting time of $60 \mu \mathrm{s}$, the ancilla qubit can be treated in a thermal equilibrium state. For an initial $|e\rangle$ state of the ancilla, the following channel simulation also works well except that the probability causing the photonic qubit decay changes from $\sin ^{2} \theta$ to $\cos ^{2} \theta$ due to the exchange of $|g\rangle$ and $|e\rangle$.

During the parity-type protocol, random decay of the ancilla qubit from $|e\rangle$ to $|g\rangle$ [with a probability of $p_{1}=\left(\pi / \chi_{\mathrm{s}}\right) / T_{1}$ ] changes the photonic qubit damping probability to $\sin ^{2}(\theta / 2)$ since only the second $X_{-\theta}$ is effective on the ancilla. The ancilla decay process could also happen with a probability $p_{2}$ during the measurement and the following waiting time. Then the adaptive $\pi$ rotation will flip the ancilla to $|e\rangle$ and mess up with the final GRAPE pulse to flip the photonic qubit from $|1\rangle$ to $|0\rangle$. Since the photonic qubit state is unknown and we simply treat this process causes a damping with a probability of $1 / 2$ (completely mixed photonic qubit state). These processes will reduce the probability of damping the photonic qubit, and based on a probability calculation we finally get the damping probability limit $\gamma^{\lim }$ the channel:

$$
\begin{aligned}
\gamma^{\lim } & =\left(1-n_{\mathrm{th}}\right)\left\{\left[1-p_{1} \sin ^{2}(\theta / 2)\right]\left(1-p_{2}\right) \sin ^{2} \theta+\left[1-p_{1} \sin ^{2}(\theta / 2)\right] p_{2} / 2+p_{1} \sin ^{2}(\theta / 2) \sin ^{2}(\theta / 2)\right\} \\
& +n_{\mathrm{th}}\left\{\left[1-p_{1} \cos ^{2}(\theta / 2)\right]\left(1-p_{2}\right) \cos ^{2} \theta+\left[1-p_{1} \cos ^{2}(\theta / 2)\right] p_{2} / 2+p_{1} \cos ^{2}(\theta / 2) \sin ^{2}(\theta / 2)\right\}
\end{aligned}
$$

Then we can get the upper limit of the external and controllable damping rate in our channel simulation:

$$
\Gamma_{1}^{\lim }=-\frac{\ln \left(1-\gamma^{\lim }\right)}{\tau_{0}},
$$

which is used for Fig. 3(e) in the main text.

\section{B. Fit of process fidelity curves in the dephasing and damping channel simulations}

The channel simulation results presented in the main text are obtained with a waiting time $60 \mu \mathrm{s}\left(\tau_{0} \approx 61 \mu \mathrm{s}\right)$. To see the channel behavior in a short time scale, we also perform simulations with a waiting time $5 \mu \mathrm{s}$ [Figs. S5(c) and (g)]. The channel process fidelity, defined as the overlap of the measured $\chi$ matrix and $\chi_{\mathrm{I}}$ for the identity operation, decays exponentially when $\theta$ is small, but deviates from the exponential behavior when $\theta$ is large. In addition, we perform the experiment with $\theta>90^{\circ}$ as well, which corresponds to a phase-flip probability $p_{\phi}>1 / 2$ for the dephasing channel [Fig. S5(d)] or $-1 \leq \sqrt{1-\gamma} \leq 0$ for the damping channel [Fig. S5(h)]. In these two cases, the process fidelity oscillates after each extra channel repetition.

In order to understand the decay and oscillation, and to verify the channel performance, we can do full numerical simulations and compare the results with the experimental data. To save time, we only numerically simulate the $\chi$ matrices of two processes 
with QuTip in Python [18, 19]: free evolution of the system for $60 \mu$ s and $5 \mu$ s respectively; and treat the channels as ideal ones because of the large number of different channels. Then, for different input states we can simply interleave the corresponding free evolution process and the ideal channel (dephasing or damping) for $n$ times to get the final states, and in turn the total process fidelity $\chi_{\theta}(t)$ at different times. We finally fit the experimental data with $F_{\chi}(t)=A\left[\operatorname{Tr}\left(\chi_{\mathrm{I}} \chi_{\theta}(t)-0.25\right]+0.25\right.$, where $A$ is a pre-factor to take into account the reduction due to the encoding and decoding processes in the experiment but not in the simulation, and 0.25 is final saturation value in the long time limit. The fitted results are shown in Fig. S5 connected with dashed lines to guide the eye, in excellent agreement with the experiment. The extracted $\theta$ 's are plotted in Fig. S6, which also agree well with the externally controlled rotation angle in the experiment.

* These two authors contributed equally to this work.

$\dagger$ clzou321@ustc.edu.cn

$\ddagger$ luyansun@tsinghua.edu.cn

[1] M. Reagor, H. Paik, G. Catelani, L. Sun, C. Axline, E. Holland, I. M. Pop, N. A. Masluk, T. Brecht, L. Frunzio, M. H. Devoret, L. I. Glazman, and R. J. Schoelkopf, "Ten milliseconds for aluminum cavities in the quantum regime,” Appl. Phys. Lett. 102, 192604 (2013).

[2] M. Reagor, W. Pfaff, C. Axline, R. W. Heeres, N. Ofek, K. Sliwa, E. Holland, C. Wang, J. Blumoff, K. Chou, M. J. Hatridge, L. Frunzio, M. H. Devoret, L. Jiang, and R. J. Schoelkopf, “Quantum memory with millisecond coherence in circuit qed,” Phys. Rev. B 94, 014506 (2016).

[3] M. Hatridge, R. Vijay, D. H. Slichter, J. Clarke, and I. Siddiqi, "Dispersive magnetometry with a quantum limited SQUID parametric amplifier," Phys. Rev. B 83, 134501 (2011).

[4] T. Roy, S. Kundu, M. Chand, A. M. Vadiraj, A. Ranadive, N. Nehra, M. P. Patankar, J. Aumentado, A. A. Clerk, and R. Vijay, "Broadband parametric amplification with impedance engineering: Beyond the gain-bandwidth product," Appl. Phys. Lett. 107, 262601 (2015).

[5] A. Kamal, A. Marblestone, and M. H. Devoret, "Signal-to-pump back action and self-oscillation in double-pump Josephson parametric amplifier," Phys. Rev. B 79, 184301 (2009).

[6] K. W. Murch, S. J. Weber, C. Macklin, and I. Siddiqi, “Observing single quantum trajectories of a superconducting quantum bit," Nature 502, 211 (2013).

[7] L. Hu, Y. Ma, W. Cai, X. Mu, Y. Xu, W. Wang, Y. Wu, H. Wang, Y. Song, C. Zou, S. M. Girvin, L.-M. Duan, and L. Sun, "Demonstration of quantum error correction and universal gate set on a binomial bosonic logical qubit," arXiv:1805.09072 (2018).

[8] M. A. Nielsen and I. L. Chuang, Quantum Computation and Quantum Information (Cambridge Univ. Press, 2000).

[9] R. Bhandari and N. A. Peters, "On the general constraints in single qubit quantum process tomography," Sci. Rep. 6, 26004 (2016).

[10] H. J. Carmichael, "Statistical methods in quantum optics 1: Master equations and fokker-planck equations (theoretical and mathematical physics)," (2003).

[11] F. Petruccione and H. Breuer, The theory of open quantum systems (Oxford University Press, 2002) p. 625.

[12] C. Gardiner, P. Zoller, and P. Zoller, Quantum noise: a handbook of Markovian and non-Markovian quantum stochastic methods with applications to quantum optics, Vol. 56 (Springer Science \& Business Media, 2004).

[13] S. Krastanov, V. V. Albert, C. Shen, C.-L. Zou, R. W. Heeres, B. Vlastakis, R. J. Schoelkopf, and L. Jiang, "Universal control of an oscillator with dispersive coupling to a qubit," Phys. Rev. A 92, 040303 (2015).

[14] M. Kliesch, T. Barthel, C. Gogolin, M. Kastoryano, and J. Eisert, “Dissipative Quantum Church-Turing Theorem,” Phys. Rev. Lett. 107, 120501 (2011).

[15] R. Sweke, I. Sinayskiy, and F. Petruccione, "Simulation of single-qubit open quantum systems," Phys. Rev. A 90, 022331 (2014).

[16] D. S. Wang, D. W. Berry, M. C. De Oliveira, and B. C. Sanders, "Solovay-kitaev decomposition strategy for single-qubit channels," Phys. Rev. Lett. 111, 130504 (2013).

[17] M. M. Wilde, Quantum Information Theory (Cambridge University Press, 2013).

[18] J. R. Johansson, P. D. Nation, and F. Nori, "Qutip: An open-source python framework for the dynamics of open quantum systems," Comp. Phys. Comm. 183, 1760 (2012).

[19] J. R. Johansson, P. D. Nation, and F. Nori, "Qutip 2: A python framework for the dynamics of open quantum systems," Comp. Phys. Comm. 184, 1234 (2013). 

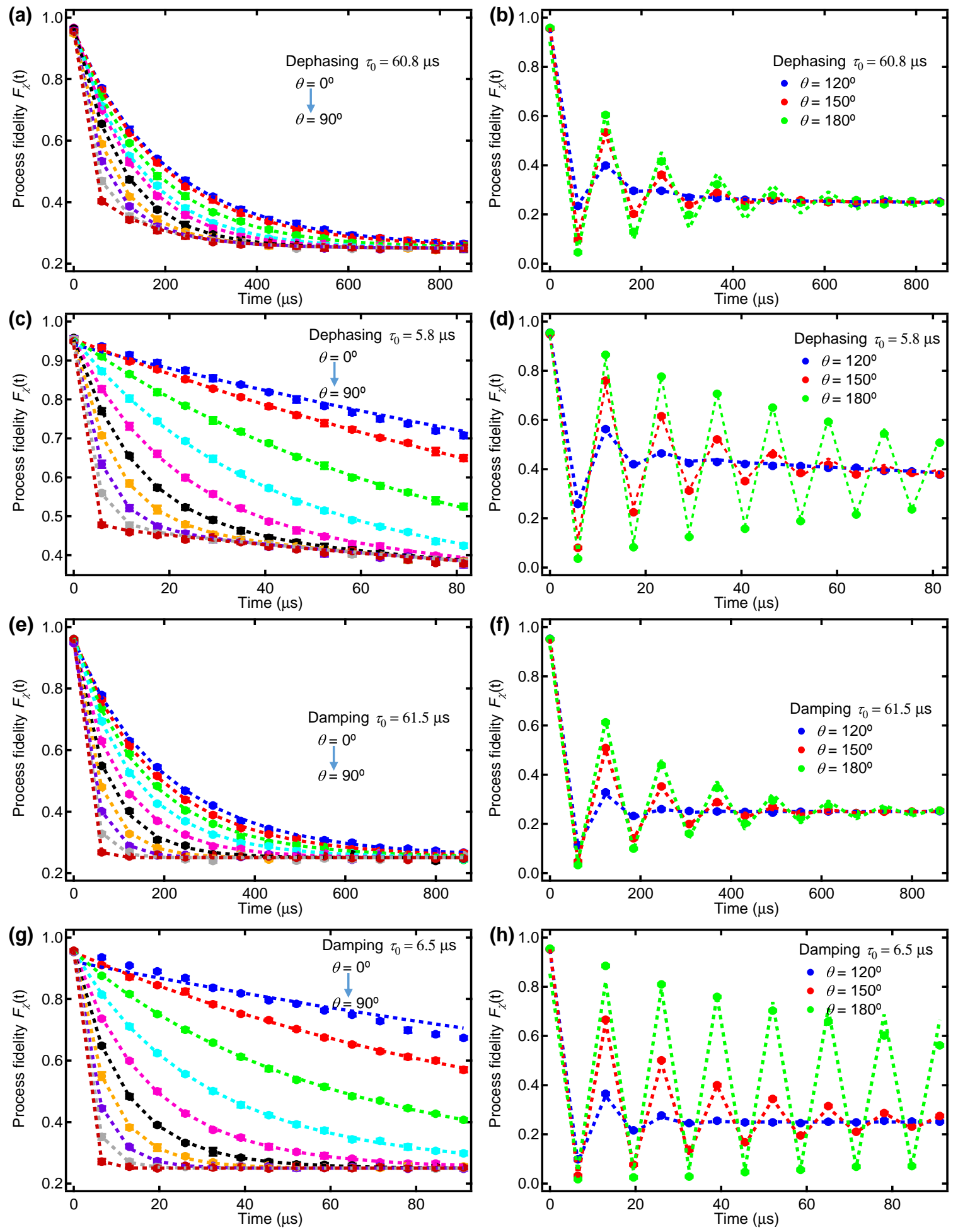

FIG. S5. Fit process fidelity curves with simulated $\chi$ matrices. We do numerical simulations with QuTip to compare with the experimental data. To save time, we only numerically simulate the $\chi$ matrices of two processes: free evolution of the system for $60 \mu$ s and $5 \mu$ s respectively; and treat the channels as ideal ones because of the large number of different channels. Due to the repetitive nature of the channel, we then simply interleave the corresponding free evolution process and the ideal channel (dephasing or damping) for $n$ times to get the total process matrix $\chi(\theta)$ at different times. Finally, the experimental data are fitted with $F_{\chi}=A\left[\operatorname{Tr}\left(\chi_{\mathrm{I}} \chi(\theta)-0.25\right]+0.25\right.$, where $\chi_{\mathrm{I}}$ is for an identity channel, $A$ is a pre-factor to take into account the reduction due to the encoding and decoding processes in the experiment but not in the simulation, and 0.25 is final saturation value in the long time limit. The fitted results are connected with dashed lines to guide the eye and are in excellent agreement with the experiment. 


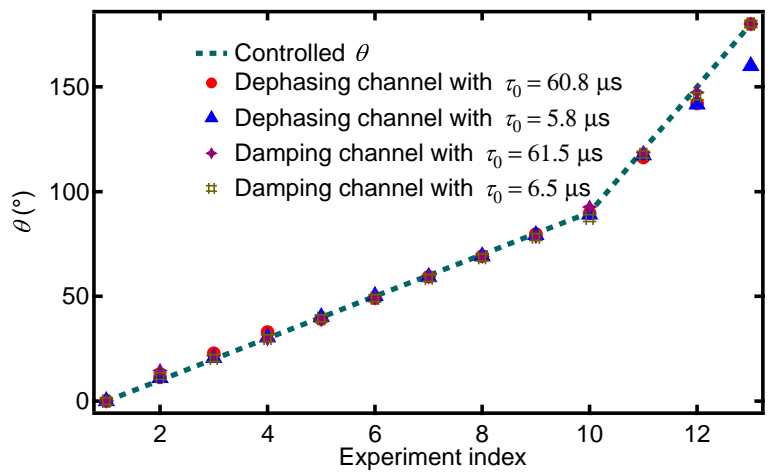

FIG. S6. $\theta$ extracted from fit of the process fidelity curves in Fig. S5. Points are the extracted $\theta$ (12 of them), in good agreement with the externally controlled rotation angles in the experiment (connected with a dashed line). 